\title{
ANÁLISIS DE OFERTA Y DEMANDA DE LOS PROGRAMAS DE ACTIVIDADES ACUÁTICAS. PROPUESTA DEL AYUNTAMIENTO DE YECLA (MURCIA)
}

Isaac Abel Martínez Palao y Francisco Disla ${ }^{2}$

${ }^{1}$ Universidad Miguel Hernández de Elche

${ }^{2}$ Ayuntamiento de Yecla

\section{OPEN ACCES \\ Correspondencio: Isaac Martínez Palao Servicio Municipal de Deportes \\ Plaza Mayor, s/n Yecla, Murcia C.P.: 30510 \\ isaac.martinez@goumh.umh.es}

Funciones de los autores: Todas las funciones fueron realizadas por los dos autores.

Recibido: $21 / 10 / 2018$ Aceptado: 01/02/ 2019 Publicado: 30/09/ 2019

Citación

Martínez-Palao, I. A., \& Disla, F. (2019). Análisis de oferta y demanda de los programas de actividades acuáticas. Propuesta del Ayuntamiento de Yecla.

RIAA. Revista de Investigación en

Actividades Acuáticas, 3(6), 60-72. https://doi.org/10.21134/riaa.v3i6.1834

@creative

(c) (1)(3) (2)

Creative Commons License Esta obra está bajo una licencia de Creative Commons ReconocimientoNoComercial-Compartir-Igua 4.0 Internacional
Resumen

s. Queda claro que la actividad física en el medio acuático está definida dentro de las más predilectas según los últimos avances dedicados al análisis de la demanda deportiva de la población española. No obstante, también es cierto que es necesario investigar que orientación es la más acertada en cuanto a la oferta de estas actividades,

deacuerdo a lo que pueda o no interesar a nuestra población, para asi poder prever las futuras necesidades y permitir ofrecer un servicio de calidad.
objetivos: El objetivo principal de esta investigación ha sido identificar estos intereses de cara a presentar una propuesta de actividades en el medio acuático encaminada a satisfacer las necesidades de todo el colectivo presente en la ciudad de Yecla, analizando el contexto en relación a la oferta y la demanda de las actividades acuáticas establecidas en la localidad.

Método: Mediante el uso de dos herramientas creadas específicamente para cada colectivo, según el nivel de participación en programas acuáticos ofrecidos por el órgano local, se

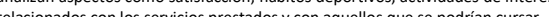

Resultados: Se encuentran numerosos resultados en los que cabe destacar que las actividades más demandadas acaban siendo el fitness acuático principalmente, y la natación estas de lunes a jueves, según determinan. En cuanto a los motivos que les llevan a la práctica de estas actividades, destacan en primer lugar la salud, seguido del desarrollo de la

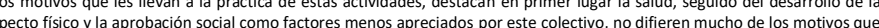
llevan a la práctica de cualquier otro tipo de actividad. Aparecen como elementos de mayor satisfacción aquellos relacionados con el personal de la instalación, siendo los vestuarios de la misma los que obtienen menor puntuación en cuanto al grado de conformidad con todo lo analizado. Se encuentran pequeñas diferencias en cuanto al sexo, la edad, y el nivel de estudios, como puede ser una menor puntuación registrada en diferentes variables cuanta mayor formación existe, o variaciones en los motivos de práctica según la edad contemplada.

Conclusiones: Partiendo de la base de estos resultados se plantean algunas indicaciones para elaborar un programa de actividades acuáticas ajustadas al interés actual de la población, sugiriendo atender las deficiencias detectadas y mejorar el servicio en aquellos aspectos que quedan al descubierto, como puede ser la evaluación y la planificación docente, la subbe enfoque al que

Title: Supply and demand analysis of water activity programs. Proposal of the city council of yecla (Murcia)

Abstract:

Background: It is clear that physical activity in the aquatic environment is defined within the most preferred according to the latest advances dedicated to the analysis of the sports according to what may or may not interest our population, in order to foresee future needs and to offer a quality service.

objectives: The main objective of this research has been to identify these interests in order to present a proposal for activities in the aquatic environment aimed at satisfying the needs of the whole group present in the city of Yecla, analysing the context in relation to the supply and demand of the aquatic activities established in the town.

Method: Using two tools created specifically for each group, according to the level of participation in water programs offered by the local body, we analyzed aspects such as satisfaction, sports habits, activities of interest and their time preferences, the motivations towards practice and some teaching and management aspects related to the services provided and those that could be studied.

Results: Numerous restls are found in which it is important to emphasize that the most demanded activities end up being mainly aquatic fitness, and educational and therapeutic swimming as activities with a greater preferential inclination. There are also three time bands marked as being of interest to attend these activities, giving rise to these from Monday belongin, a docit practice of any other type of activity. Those related to the facility's personnel appear as elements of greatest satisfaction, with the changing rooms obtaining the lowest score in terms of the degree of compliance with everything analysed. Small differences are found in terms of sex, age and level of studies, such as a lower score recorded in different variables for the higher training, or variations in the reasons for practice according to the age considered.

Conclusions: On the basis of these results, some indications are proposed to develop a program of aquatic activities adjusted to the current interest of the population, suggesting to attend to the deficiencies detected and to improve the service in those aspects that are revealed, such as the evaluation and the educational planning, the correction of the problems detected in the installation, the orientation that can be given to the time distribution bands, the type of activities and the approach to which to direct them. Keywords: Satisfaction, service quality, aquatic activities, aquatic programs, supply and demand, practice interests

Titulo: Oferta e exigência de análise dos programas de atividades aquáticas. Proposta do conselho da cidade de yecla (Murcia)

Resumo

Antecedentes: É evidente que a atividade física no ambiente aquático é definida entre as mais favoritas, de acordo com os últimos avanços dedicados à análise da demanda esportiva

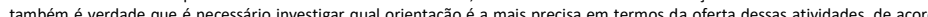
pode ou não interessar à nossa população, a fim de antecipar necessidades futuras e nos permitir oferecer um serviço de qualidade

Objetivos: $\mathrm{O}$ principal objetivo desta pesquisa foi identificar esses interesses, a fim de apresentar uma proposta de atividades no ambiente aquático, visando atender às necessidades de todo o grupo presente na cidade de Yecla, analisando o contexto em relação à oferta e a demanda das atividades aquáticas estabelecidas na localidade.

Método: Através do uso de duas ferramentas criadas especificamente para cada grupo, de acordo com o nível de participação nos programas aquáticos oferecidos pelo órgão local são analisados aspectos como satisfação, hábitos esportivos, atividades de interesse e preferências de tempo, motivaçōes para a prática. e alguns aspectos de ensino e gestão relacionados aos serviços prestados e aos que poderiam ser adotados. terapêutica comom inumeros resultados nos quais vale ressaltar que ás existem três faixas marcadas de interesse horário para participar dessas atividades, dando origem a eles de segunda a quinta-feira, conforme determinado. Quanto aos motivos que os levam a pratica dessas atividades, destacam-se em primeiro lugar a saúde, seguida pelo desenvolvimento da habilidade e pertencimento a um grupo social, deixando o aspecto físico e a aprovação social como fatores menos apreciados por este. coletivo, eles não diferem muito dos motivos que levam a pratica de qualquer outro tipo de atividade. Os relacionados ao pessoal da instalaça aparecem como elementos de maior satisfaçao, sendo os vestiários dos mesmos que obtêm menor pontuação em termos de grau de conformidade com tudo analisado. Existem pequenas diferenças em termos de sexo, idade e nível de educação, como

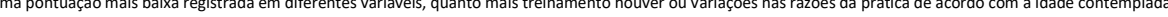

(a) a da

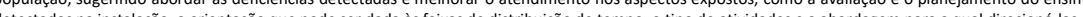
Palavras-chave: Satisfação, qualidade de serviço, atividades aquáticas, programas aquáticos, oferta e demanda, interesses de prática. 


\section{Introducción}

Según la última encuesta realizada por el Instituto Nacional de Estadística y el Ministerio de Educación, Cultura y Deporte sobre los hábitos deportivos de los españoles (2015), la natación es el segundo deporte más practicado en España con un $38.5 \%$ de la población que práctica este deporte en el último año. Además, según García Ferrando y Llopis Goig (2017), en 2014 la Comisión Europea publicó las tasas de práctica deportiva de una encuesta sobre deporte y actividad física en las que, mencionan, que España obtiene una tasa del $46 \%$ de práctica deportiva con la regularidad de al menos una vez por semana, aspecto que, dicen, deja a los españoles cinco unidades porcentuales por encima de la media de ciudadanos europeos practicantes. Apreciando todo esto y en contraste con lo que comentan, relativo al anterior Eurobarómetro en 2009, se aprecia un claro ascenso del nivel de práctica deportiva española. Asimismo, exponen en sus resultados que la natación, con una tasa de casi un $20 \%$, se encuentra entre los cuatro deportes más practicados en España actualmente.

En línea con esto, Sánchez, González, López, y Díaz, (2017) declaran que hoy en día, las actividades acuáticas están en apogeo debido a la demanda existente en la población, indican también, que la satisfacción de los usuarios es un elemento imprescindible para evitar que los niveles de ejercicio físico no desciendan. Por otro lado, Martín, González, Campos, Del Hierro, y Jiménez-Beatty (2010) recogen la natación y las actividades acuáticas como predilectas entre los adultos mayores que no practican, pero si les gustaría practicar ejercicio físico. Plantearon que había numerosos estudios que recogían datos sobre hábitos deportivos y las actividades que más se practicaban, pero no lo hacían sobre las personas que no practicaban, pero sí querrían hacerlo, haciendo referencia al termino que utilizan en este aspecto a la "demanda latente". En su estudio, analizaron las actividades más demandadas por este colectivo y también las expectativas de benéficos esperadas por el mismo. Además, remiten acerca de resultados similares de varios documentos, en los que el contexto en cuanto a oferta de servicios, les permitió esa comparación.

Teniendo en cuenta lo anterior, otros estudios como el de Tuero y González-Boto, (2015) destacan la importancia de ahondar en el conocimiento del cliente de las instalaciones acuáticas, debido principalmente a que el aumento de actividades ofertadas en estas y la demanda que generan, insta a reconocer las conductas, necesidades y motivos de práctica de los mismos, para así poder obtener datos objetivos que faciliten a los gestores la toma de sus decisiones. Según ellos, "resulta un aspecto básico comprobar si las instalaciones responden a las necesidades del público usuario", confirmando la necesidad del uso de herramientas apropiadas que permitan extraer las posibles contribuciones y percepciones de los usuarios, ya que estas repercutirán a la hora de ofertar los servicios en las instalaciones acuáticas de forma óptima.

De este modo, el análisis de las opiniones de los usuarios puede darnos importantes datos de manera objetiva, siendo esto una base fundamental que, no solo facilite la toma de decisiones sobre la oferta de los servicios, si no, que además nos permite prever la futura demanda y mejorar el servicio. (Armada, Martínez-Gallego, Segarra, y Díaz, 2016). Los autores remarcan que el conocer estas opiniones, es la clave para la fidelización y un gran paso para adquirir una fórmula que permita dar a los ciudadanos un "valor añadido, que sea de calidad".

"En el plano de la conciencia directa, cada vez que el usuario recibe un producto o servicio se produce un juicio acerca de la calidad del mismo" (Medina Rodríguez, 2010). La autora, explica que este juicio de calidad, puede llevarse a lo racional mediante el apoyo de un proceso de interrogación y reflexión, cuyo fruto puede darnos la información necesaria para conocer el grado y el contenido del servicio deseado, especifica que la mejor fuente de investigación para lograrlo, puede ser el usuario. Siguiendo esta línea, hace referencia a que existe una constante evolución del deporte en el ámbito social, y la necesidad de poder adaptarse a ella, conlleva que los procesos de gestión de las entidades deban prestar atención a las exigencias y necesidades de los usuarios. De esta forma, dice, las entidades que utilicen sistemas de gestión con un enfoque de calidad, lograrán mayor posibilidad de desarrollarse y ajustarse a las exigencias del sector.

Para poder abordar esta necesidad y adaptarnos a esta constante evolución, como bien comentaban anteriormente Tuero y GonzálezBoto (2015), se hace imprescindible el uso de algún instrumento que nos permita esclarecer todos los aspectos relevantes que posibiliten el hecho de ofrecer actividades más ajustadas a cada sección de población y saber exactamente donde y cuando ubicar cada servicio. Ya que como remarcan, los resultados de ciertas variables pueden revertir directamente en la gestión y el funcionamiento de las piscinas cubiertas. Es por esto, que exponemos a continuación, algunas de estas variables que se analizan en este tipo de estudios, con el fin de conocer mejor que características se han de tener en cuenta y son necesarias de cara a poder elaborar, de la mejor forma, nuestro trabajo. En su estudio analizan variables sociodemográficas, sobre hábitos y motivaciones en cuanto a la práctica, y relacionadas con la gestión y organización de las actividades realizadas, recogiendo también información relativa a los márgenes horarios con mayor o menor satisfacción, distribuida en segmentos de edad, y algunos aspectos relacionados con las preferencias de actividad por género.

Otros estudios más recientes evalúan la satisfacción que tienen los usuarios en relación a toda la gestión de la misma, incluyendo aspectos relacionados con las instalaciones, material, comunicación, organización, monitores, calidad - precio y actividades realizadas (Sánchez et al., 2017). Su objetivo fue diagnosticar las debilidades más generales, para así reducir el déficit entre los servicios ofertados y las expectativas del usuario. En la misma línea se mueven García, Vergara, López y Díaz (2016), que se centran en evaluar la calidad percibida y la satisfacción de los usuarios con las organizaciones que prestan los servicios deportivos, determinando aspectos como son la imagen de la organización, instalación, material, tipos de actividades y aspectos técnicos generales.

Uno de los trabajos más destacados en el tema que nos ocupa es el de Moreno (1997), en este extenso documento, entre otras cosas, se analizan por completo los grandes aspectos relacionados con la gestión deportiva en instalaciones acuáticas, localizando el foco en tres grandes áreas de observación, como son los usuarios, los educadores y los gestores de instalaciones acuáticas cubiertas, dando como resultado el análisis de factores que permiten conocer la demanda de actividades acuáticas y su nivel de satisfacción con las mismas, información acerca de la oferta de servicios y si estos coinciden con las expectativas de los gestores, y aspectos relacionados con las características metodológicas y los recursos didácticos que se dan en los programas.

Por último, en un reciente estudio en México, Álvarez-Fariña (2018) analiza la oferta de programas acuáticos que existía por parte de las empresas en 131 instalaciones acuáticas, y la demanda que generaban, según el tipo de programas que ofrecían. En él, de 23 empresas que respondían a estas características, 13 daban datos acerca de los tipos de programas, 9 estaban enfocadas a programas con actividades con un corte metodológico de aspecto técnicodeportivo, buscando el rendimiento y el sentido utilitario, y 4 aplicaban programas relacionados con metodologías encauzadas de forma lúdica, teniendo en cuenta perspectivas más educativas, cognitivas y socio-emocionales. En sus resultados, los programas con la orientación metodológica de características recreativas que 
consideraban valores más educativos, obtuvieron una gran diferencia en cuanto al número de inscritos, la demanda de este tipo de actividades fue de un $72 \%$ frente a un $28 \%$ de las propuestas con un tipo de enseñanza más técnica y utilitaria. El autor concluye que, en su conjunto, los resultados obtenidos dejaron ver una disonancia entre la oferta de actividades en México y la demanda generada en las mismas, menciona que es necesario reflexionar sobre las estrategias didácticas y los tipos de metodologías que predominan en la enseñanza de la natación.

Como podemos apreciar hay gran diversidad de posibles variables que contemplar, pero más importante es el efecto que pueden llegar a tener en el éxito de la aplicación del tipo de programa ofrecido, en consonancia con lo que realmente busque la sociedad, llegando incluso a afectar a una nación entera, como hemos visto en este último caso.

De esta forma, entendemos que es pertinente analizar el contexto en el que se enmarca la población de Yecla, analizando variables como satisfacción, motivos de práctica, actividades de interés, franjas horarias y días de preferencia, aspectos relacionados con la forma de dar las clases, y demás factores que tengan que ver con los intereses y preocupaciones de todos los ciudadanos de esta localidad en cuanto a actividades acuáticas se refiere. Fijando como objetivo contrastar la demanda que surja, con la oferta de programas actuales y, finalmente, ofrecer un programa acorde al interés real de la ciudad.

Para ello en este trabajo se pretende analizar, con la colaboración de la Asociación Iberoamericana de Enseñanza y Terapia Acuática (AIDEA) y el Excmo. Ayto. de Yecla, el contexto de la población de este municipio respecto a los programas de actividades acuáticas, comprobando la relación entre la oferta y la demanda, identificando las necesidades y preocupaciones de los usuarios y observando si hay intereses nuevos, o la demanda se asemeja a lo que la ciencia nos dice en cuanto a que tipos de programas funcionan en otras poblaciones con dimensiones similares o mayores, y poder así ofertarlos de igual forma.

\section{Método}

\section{Participantes}

Como ya hemos mencionado, la investigación se llevó a cabo en la localidad de Yecla, analizando las preocupaciones e intereses de la misma en relación a la instalación acuática allí constituida. El público objetivo del estudio fueron los usuarios y los no usuarios de la instalación acuática cubierta, perteneciente a la administración local. Entendiendo como usuarios, según Moreno (1997), a aquellas personas que asisten o hacen uso en la instalación de alguno de los programas de actividad físico-deportiva en el medio acuático, establecidos por la administración, encontramos que la muestra de estos usuarios, está constituida por un total de 338 sujetos, distribuidos en un $35,8 \%$ hombres y un $64,2 \%$ mujeres.

Dicha muestra se situó entre los 6 meses y los 81 años de vida, siendo las respuestas de los menores de edad, recogidas por mediación de un adulto a su cargo. La edad media de la muestra fue 37 años, siendo la relación de edad media y genero, 35 años en varones, y 38 en mujeres. Esta muestra quedo seccionada en 4 franjas de edad, siendo el $18 \%$ menores de 24 años la primera franja, el $24 \%$ de 25 a 35 años la segunda, el $45 \%$ de 36 a 54 años la tercera, y el 13\% contemplando a todos los mayores de 54 años en la última franja de edad.

En cuanto al nivel de estudios el 40\% llegaba a tener estudios superiores y el resto estaba distribuido en un 39\% de estudios medios y secundarios y un $21 \%$ primarios. Con una situación de ocupación de un $18 \%$ que estudiaba, un $60 \%$ trabajaba, el $11 \%$ en paro y un $10.9 \%$ dedicado a las labores del hogar y jubilados.
La muestra de los sujetos no participantes en dichos programas o "no usuarios", estuvo compuesta por un total de 266 personas de entre 16 y 75 años, de forma que el sexo quedó dividido en el $23 \%$ para los hombres y el $77 \%$ para las mujeres. La selección muestral, quedó repartida en la misma maya de población que abarcó los 4 grupos de edad mencionados anteriormente, quedando 33 sujetos menores de 24 años, 71 de entre 25 y 35 años, 99 sujetos de 36 a 54 años y 47 individuos de más de 54 años, dejando los 16 restantes, que no determinaron su edad.

El nivel de estudios de esta muestra se vio distribuido en un $37,6 \%$ de personas que poseían estudios superiores, un $43,8 \%$ de estudios medios y secundarios, y un $18,6 \%$ de estudios primarios, siendo los porcentajes de ocupación, por entonces, un 53,8\% de personas que trabajaban, un $13,5 \%$ de ellas estudiaban, un $12,4 \%$ respectivamente, estaban en paro o jubiladas y el $8 \%$ dedicadas a labores del hogar.

\section{Medidas}

A la hora de desarrollar el instrumento, en primer lugar, fue imprescindible un análisis previo de diferentes fuentes relacionadas con los aspectos que nos ocupaban. Para poder abordar la mayor parte de los intereses de la población y asegurar la recogida de todas las variables que pudieran albergar datos relativos a la demanda de la misma, se hizo necesario el diseño de dos cuestionarios (Anexos I y II) para el registro de estos, un cuestionario exclusivo para analizar los intereses y la demanda de los usuarios participantes en los servicios de la piscina cubierta (C.A.I.D.U.), y otro para aquellos que no hacían uso de los mismos (C.A.I.D.N.U.). Ambos contienen ítems de diferentes formatos, existen algunas preguntas de carácter abierto, pero predominan las que son de carácter cerrado, esto fue así, para facilitar el tiempo de respuesta y evitar el aspecto tedioso que podía suponer lo contrario. En estas últimas, la formulación es variada, podemos encontrar ítems con respuesta alternativa, de selección múltiple, con casillas de verificación, y cuadrículas de respuesta múltiple o de verificación.

En cuanto a las dimensiones que constituyen ambas herramientas, se utilizó como base el cuestionario para el análisis de la demanda de los programas acuáticos (CADPA) de Moreno (1997), adaptando algunas de estas áreas a los intereses que se nos prestaban para el estudio, e incorporando nuevas, extraídas de fuentes algo más actuales.

Aspectos sociodemográficos. Los datos que reúne esta área tienen que ver con las características de los usuarios y su entorno personal. Recoge variables como el sexo, la edad, nivel de estudios, situación laboral, número de hijos y sector de residencia.

Intereses de práctica. Este bloque contempla variables que describen los intereses de cara a diferentes tipos de programas y aspectos relacionados con la gestión de los mismos. Esta distribuido en varios apartados en función de la participación del sujeto. En el CAIDU, recoge la orientación del interés hacia determinadas actividades y solicita de forma libre cualquier cuestión a tener en cuenta sobre las mismas. Además de estas, el CAIDNU también atiende aspectos relacionados con los posibles horarios y días de asistencia, y extrae la información conocida sobre los programas actuales.

Motivaciones de práctica. Para medir las razones que mueven hacia la práctica de ejercicio físico y actividades acuáticas a los sujetos, se ha utilizado el cuestionario de contenido de los objetivos en el ejercicio (GCEQ) de Sebire, Standage y Vansteenkiste (2008), cuestionario que fue traducido y validado al español por Moreno-Murcia, Marcos-Pardo y Huéscar (2016). Mediante una escala de respuesta Likert de 7 puntos, que mide la intensidad de la importancia que les dan los sujetos a las variables de reconocimiento social, imagen, salud, 
desarrollo de habilidad y afiliación social, se preguntan un total de 20 ítems distribuidos por igual en estas 5 variables.

Hábitos deportivos. En esta dimensión se plantean cuestiones sobre tiempo y frecuencia de práctica de aquellos que, si practican ejercicio físico, y motivos por los que no lo hacen en aquellos que no tengan este hábito establecido. Se pregunta si realizan actividad, de que tipo y con qué asiduidad semanal, siguiendo las indicaciones de la OMS.

Identificación del programa. Recopila todos los datos que definen la situación del usuario en cuanto a los programas y conocimiento que tienen sobre los mismos. Se distribuye en 3 apartados claramente marcados, programa en el que participa, como lo descubrió y cuáles de las actividades que se ofrecen, realmente sabe en qué consisten.

Evaluación docente. Área que mide la calidad de la intervención de monitor/a mediante una adaptación del cuestionario de MorenoMurcia, Silveira y Belando (2015) de evaluación de las competencias docentes en el ámbito universitario. El cuestionario original de evaluación de la intervención docente (CEID) de estos autores, tuvo que ser adaptado a un ámbito acuático en el que se relacionaron los ítems con aspectos de una instalación acuática y sus monitores, modificando algunos de los ítems a un lenguaje más coloquial y aplicado a este medio, y eliminando aquellos que no iban en relación a términos que nos fuesen de interés en el mismo (e.g. "Incorpora y emplea las TICs" o "Es fácilmente accesible a tutorías", etc.). La versión final quedó formulada por 19 ítems agrupados en 3 variables: Planificación, Desarrollo y Resultado, dejando tres ítems en la primera, diez en la segunda y seis en la tercera, con el rigor de no dejar sin consistencia el cuestionario. La valoración de estos ítems, viene dada por una escala Likert de 5 puntos con el criterio de adhesión de 1 totalmente en desacuerdo y 5 totalmente de acuerdo.

Satisfacción General. Cuestionario extraído directamente del CADPA de Moreno (1997), validado y constituido por 22 ítems, que extraen donde pueden erradicar los posibles problemas en el desarrollo de los programas o el uso de la instalación, si los hubiese. Enmarca 4 grandes factores (personal de la instalación, condiciones higiénicas y de mantenimiento, vestuarios y espacios de la instalación, y aspectos relacionados con la gestión y facilidades de acceso a la misma) que son valorados con una escala de 4 puntos en la que 1 es nada satisfecho y 4 totalmente satisfecho.

\section{Procedimiento}

En el momento en el que se plantea llevar a cabo este documento, se procede, en instancia primera, a revisar la literatura científica en relación al tema que abordamos. De este modo quedó claro el modelo a seguir y partiendo de un diseño de dos herramientas para medir los aspectos que nos ocupaban, se dio lugar el proceso de recogida de la información.

Al ser un estudio de corte transversal, llevado a cabo en el último trimestre de la temporada de servicios, contemplada entre los meses de marzo, abril y mayo del año 2019, y coincidir con la apertura del nuevo vaso en la instalación, dio lugar a que el proceso se planteara buscando la mayor celeridad en los resultados, dejando como opción más viable en el caso de los sujetos no participantes en ningún programa acuático, tener que realizar un muestreo intencionado en diferentes instituciones públicas y privadas, así como personas afines y conocidas de otros servicios.

Para el diseño muestral de los sujetos no participantes, se solicito en el padrón de la administración local, el número total de hombres y mujeres, por rangos de edad, existentes en el momento. Esto, permitió conocer exactamente cuanta cantidad de gente había por género en cada rango de edad y posteriormente discernir el tamaño exacto de la muestra necesaria para llevar a cabo un proyecto en condiciones de rigor científico. El valor que se tomó como error en cuanto al nivel de significación fue un $5 \%$, dejando claro así el número de ítems que podían estar conformes o no.

En cuanto a la recogida de información de los usuarios participantes en los programas acuáticos, se intento recopilar el mayor número de informes posibles en el tiempo que se estableció como límite para comenzar a analizar los datos y poder realizar la propuesta antes de que comenzara el nuevo periodo lectivo. Para ello, se contactó con el servicio municipal de deportes de la localidad y tras los pertinentes procesos burocráticos, se transmitió una circular a través de la directiva deportiva municipal, con la que se dio por comenzada la recolección de la información.

Esta se llevo a cabo mediante un enlace informático asignado con un código QR para cada uno de los cuestionarios, que fue distribuido por todos los integrantes del servicio de la instalación, facilitando así que llegara a la máxima población posible, utilizando los grupos de distribución de información que los monitores tenían con sus usuarios, incorporando panfletos informativos y flyers en tablones de anuncios y conserjería, y aprovechando el resto de servicios que el ayuntamiento ofrecía en el ámbito deportivo, para fomentar la distribución de los mismos. Además de esto, se generó un cartel modelo que fue colocado en todas las instalaciones públicas y se contactó con cantidad de clubs y asociaciones, todos los colegios y centros de secundaria, y se enviaron los cuestionarios y los enlaces a todas las AMPAS con las que la administración pudo lograr el contacto, todo esto con el fin de aumentar el impacto y conseguir la mayor muestra posible.

Se ha de mencionar que en todo momento los datos recogidos fueron anónimos, sin reflejar ninguna referencia a la identificación del sujeto dentro del proceso.

Por otro lado, en algunos casos, la recogida de datos se dio mediante la impresión en papel de ambas herramientas, para poder llegar a colectivos con los que se hacía más complejo el uso de las nuevas tecnologías.

\section{Análisis de datos}

Se llevaron a cabo análisis descriptivos y análisis diferenciales con $\mathrm{x}^{2}$ usando como variables independientes la edad, el sexo y el nivel de estudios mediante SPSS Statistics 22.0.

\section{Resultados}

\section{Resultados descriptivos}

A raíz del análisis de los resultados obtenidos, a continuación, se detallan algunas de las características que nos ayudan a ubicar mejor a los participantes del estudio.

Para poder hacer una correcta diferenciación, se presentan en primer lugar, los resultados de ambos grupos de sujetos, los correspondientes al cuestionario de usuarios participantes en algún programa, y los relativos al que no participan en ninguno.

En referencia al grupo de usuarios, la muestra queda distribuida en función de las actividades de las que hacen uso, siendo estas el baño libre con un $17 \%$, iniciación a la natación con un $12,6 \%$, adaptación al medio acuático con un $11,6 \%$, natación para adultos y Aquagym ambas con un $11,2 \%$, iniciación avanzada con un $10 \%$, asociaciones y clubs con un $9,6 \%$, natación terapéutica con un $4,8 \%$, bebes con un $3,7 \%$, aprendizaje de la natación con un $3 \%$, natación en jóvenes con un $1,8 \%$, actividades acuáticas para mayores con un $1,6 \%$, 
perfeccionamiento de la natación con un 1,4\% y Matronatación con un 0,5\%, (Figura 1)

Figura 1. Distribución de los usuarios de la piscina en función de las actividades que realizan.

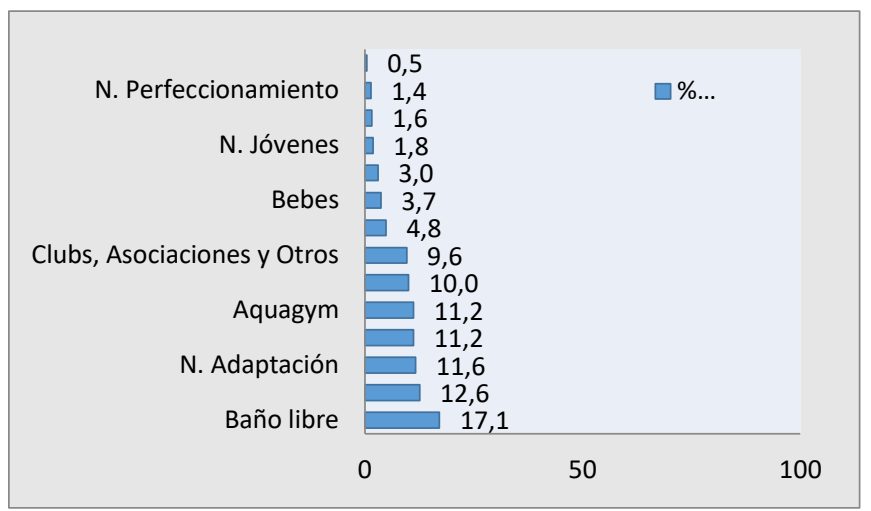

Por otra parte, de la muestra obtenida de sujetos no participantes en ninguno de los programas acuáticos ofertados, el $79 \%$ practican algún otro deporte, frente al $21 \%$ que no lo hacen, debido, principalmente a un $36,8 \%$ por falta de tiempo, un $16 \%$ por dificultad horaria, un $11,5 \%$ por falta de interés o por motivos económicos, un 10,3\% por no tener con quien asistir, un 9,2\% por falta de instalaciones cerca y un $3 \%$ por motivos de salud, como podemos apreciar en la Figura 2.

En cuanto a las actividades realizadas por ese $79 \%$ que no participa en ningún programa acuático establecido por la administración local, pero sí practica alguna otra actividad, observamos que entre las actividades más practicadas se encuentran el Yoga, Pilates o el Tai chi con un $16,5 \%$, el Running con un $13 \%$, gimnasia de mantenimiento tiene un $12,4 \%$, actividades relacionadas con el Fitness un $8,4 \%$, caminar un $8 \%$, otras actividades natatorias distintas a las ofertadas un $7,2 \%$, musculación un $6,4 \%$, actividades de montaña con un $5,2 \%$, ciclismo un $4,8 \%$, baile y futbol ambas con un $3,6 \%$ y atletismo con un $2,8 \%$, quedando un $8 \%$ restante que realizan otro tipo de actividades (Judo, karate, pádel, crossfit, actividades de indoor y clases dirigidas, suelo pélvico y algún tipo de ejercicio en casa), (Figura 3).

Además, la frecuencia de práctica de estas actividades reflejada en la Figura 4, nos detalla que el $55 \%$ practica su actividad de uno a dos días por semana, el $20 \%$ tres días por semana, el $18,4 \%$ la practica más de tres días a la semana y casi el $7 \%$ lo hace de forma esporádica.

Figura 2. Motivos por los que los sujetos no participantes en programas acuáticos no realizan actividad física.

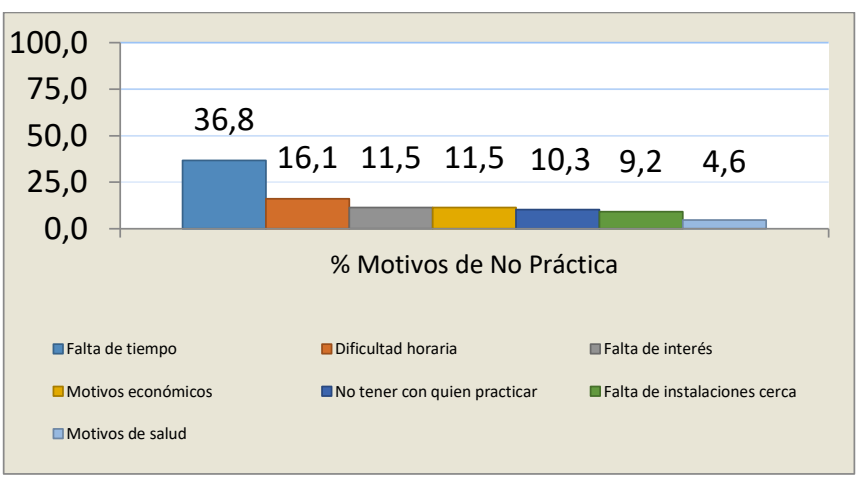

Figura 3. Otras actividades realizadas por los sujetos no participantes en ningún programa acuático ofertado.

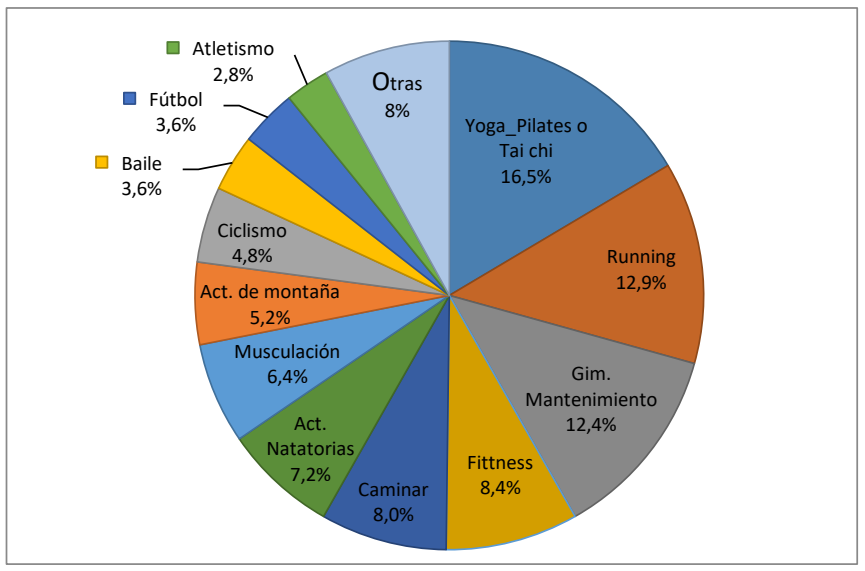

Figura 4. Frecuencia de práctica de otras actividades diferentes a las ofertadas.

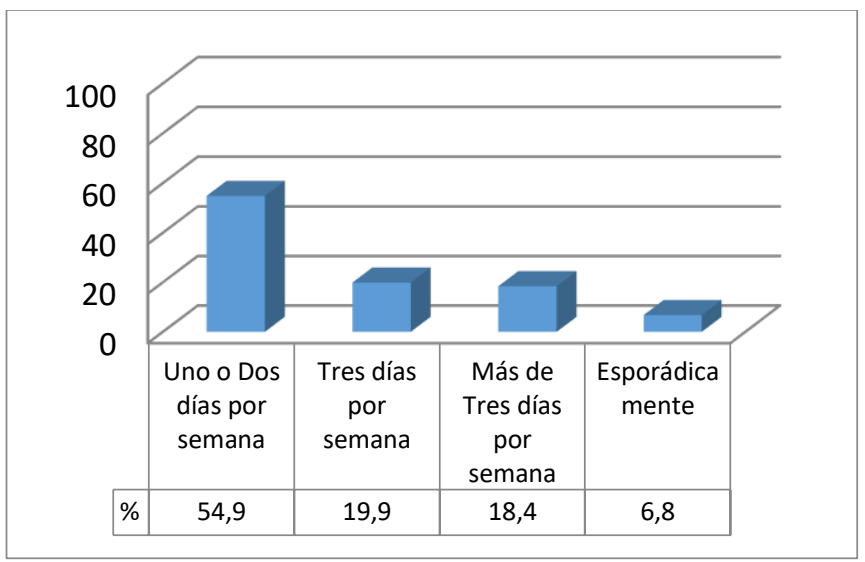

Respecto al estado de ocupación actual de los sujetos, los usuarios participantes en alguno de los programas (Figura 5) se dividen en un $60 \%$ trabajando, un $18 \%$ estudiando, un $11 \%$ en paro, un $6,3 \%$ jubilados y un $4,6 \%$ dedicado a labores del hogar, en consonancia con los sujetos no participantes en ninguno de los programas acuáticos (Figura 6), que quedan distribuidos en un 53,8\% trabajando, un $13,5 \%$ estudiando, un $12,4 \%$ jubilados o en paro y un $8 \%$ dedicado a labores del hogar.

En las Figuras 7 y 8 se expone la configuración de individuos en función de su zona de residencia, quedando, en los usuarios el $27 \%$ alojados en el ensanche oriental de la localidad, el $24,8 \%$ en el centro urbano, el $12,9 \%$ en el ensanche occidental, el $11,9 \%$ vive fuera de la localidad o en las inmediaciones de la misma, el 8,4\% reside en la zona antigua de la ciudad, el $7,7 \%$ en el contorno del barrio Alameda, el $3,9 \%$ se alberga en la zona del barrio del sol y el hospital y el 3,5\% está instalado en el paseo del cementerio y el barrio San Nicolás, mientras que la muestra obtenida de aquellos sujetos no participantes en los programas acuáticos queda repartida en un $25 \%$ residente en el centro urbano, un $23 \%$ en el ensanche oriental, un $13,5 \%$ habita en las inmediaciones locales o fuera de la misma, un $12,7 \%$ en el ensanche occidental, un $9 \%$ en el barrio alameda, un $8 \%$ en la zona antigua, un $5,7 \%$ en la zona de hospital y barrio del sol y un $3,3 \%$ por S. Nicolás y el paseo del cementerio.

Asimismo se muestra la cantidad de hijos por sujeto en la tabla 1 , siendo 2 hijos la mayoría de respuestas dadas en ambos cuestionarios con un $36,7 \%$ en los usuarios y un $32,8 \%$ en los que no lo son, seguido 
de igual forma por aquellos que no contestan o no tienen hijos con un $32,8 \%$ en ambos grupos de individuos, un $14,8 \%$ y un $17,9 \%$ respectivamente marcando un solo hijo, un $10,7 \%$ en los usuarios y un 9,5\% en los no usuarios para los 3 hijos, un 3,8\% con 4 hijos en ambos casos y un restante 1,2 y $3 \%$ para más de 5 hijos, respectivamente.

Figura 5. Ocupación actual usuarios.

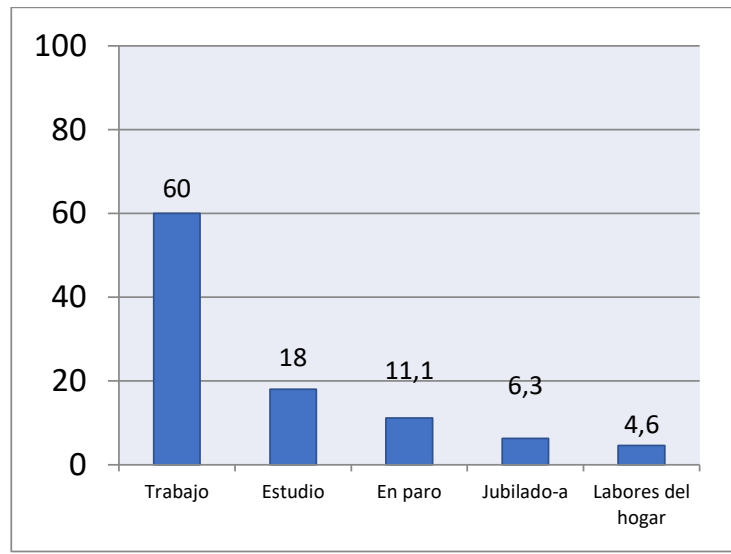

Figura 6. Ocupación actual no usuarios.

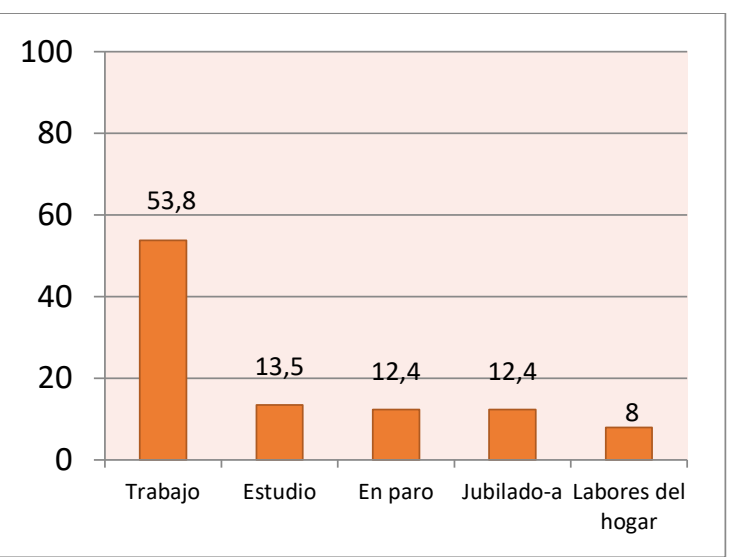

Figura 7. Distribución Usuarios por zona residencial.

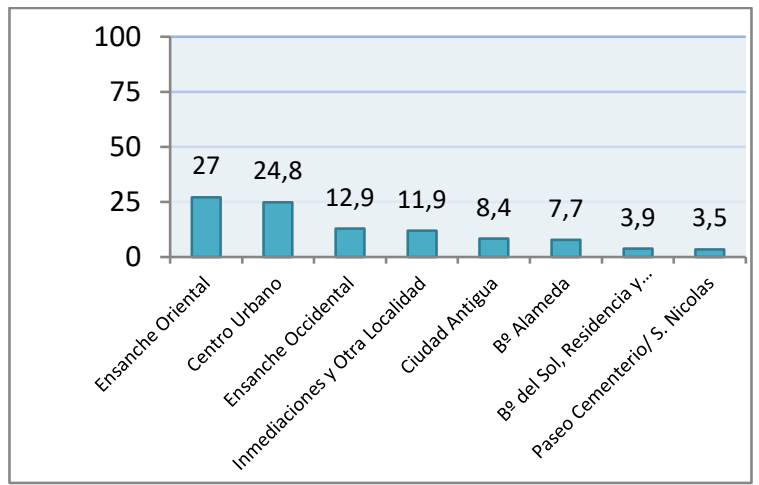

Figura 8. Distribución no usuarios por zona residencial.

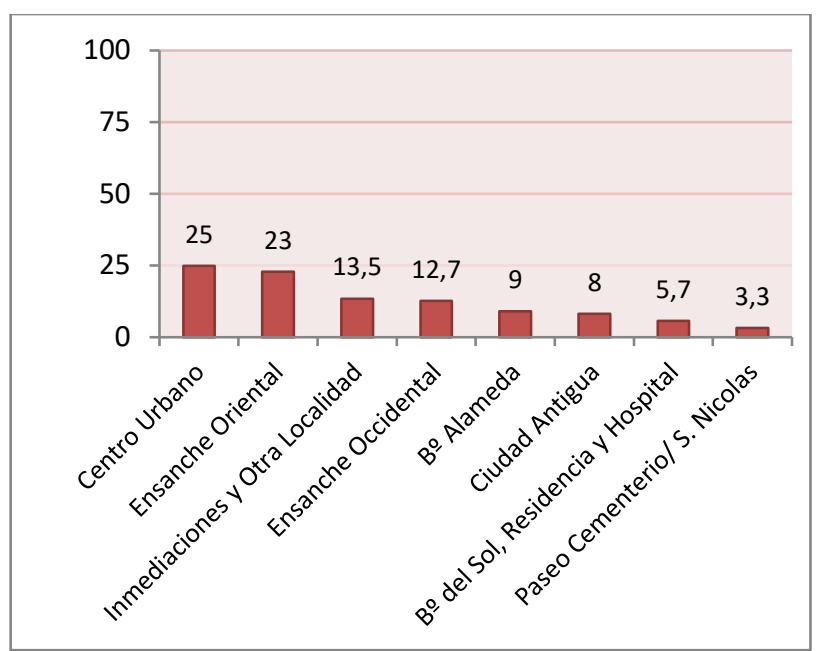

Tabla 1. Cantidad de hijos por sujeto en porcentaje.

\begin{tabular}{|c|c|c|c|}
\hline № de Hijos Usuarios & (\%) & $\begin{array}{c}\text { № de Hijos No } \\
\text { Usuarios }\end{array}$ & (\%) \\
\hline 2 & 36,7 & 2 & 32,8 \\
\hline 0 & 32,8 & 0 & 32,8 \\
\hline 1 & 14,8 & 1 & 17,9 \\
\hline 3 & 10,7 & 3 & 9,5 \\
\hline 4 & 3,8 & 4 & 3,8 \\
\hline 8 & 0,6 & 5 & 1,5 \\
\hline 7 & 0,3 & 8 & 1,1 \\
\hline 12 & 0,3 & 7 & 0,4 \\
\hline
\end{tabular}

Atendiendo al conocimiento que tienen los participantes del estudio, sobre los programas ofertados por el servicio deportivo municipal, en la Figura 9, podemos apreciar que apenas el $4 \%$ de todos los usuarios, no contestan o no conocen ninguno de los programas a los que pueden optar en cuanto actividades acuáticas se refiere, en contraposición al $96 \%$ de estos usuarios, que determinan que las actividades más conocidas son la iniciación a la natación con un 13\%, la adaptación al medio acuático con un $11 \%$ y la natación para adultos y el Aquagym con un $10 \%$, seguidas de la iniciación avanzada, el aprendizaje de la natación, el perfeccionamiento de la natación y la natación terapéutica con un $9,8,8$ y $7 \%$ respectivamente, para dejar natación para bebes y jóvenes con un $6 \%$ y actividades acuáticas para mayores y Matronatación con un 5 y un 3\% correspondientes.

De forma similar quedan distribuidas, en la Figura 10, las actividades ofertadas en aquellos individuos que no participan en ningún programa acuático, siendo algo mayor la muestra que no contesta o no conoce ningún programa (12\%) que en el caso anterior, y dejando el $88 \%$ de respuestas, repartidas igualmente en un $11 \%$ para natación para adultos e iniciación, un $10 \%$ para Aquagym y un $9 \%$ para adaptación al medio, como actividades más conocidas, continuadas por la natación terapéutica y el aprendizaje con un $8 \%$, la iniciación avanzada con un $7 \%$, y finalizando con el perfeccionamiento y las actividades para mayores con un $6 \%$, natación en jóvenes y bebes con un $5 \%$ y Matronatación con un $2 \%$. 
Figura 9. Actividades más conocidas por los usuarios de programas acuáticos.

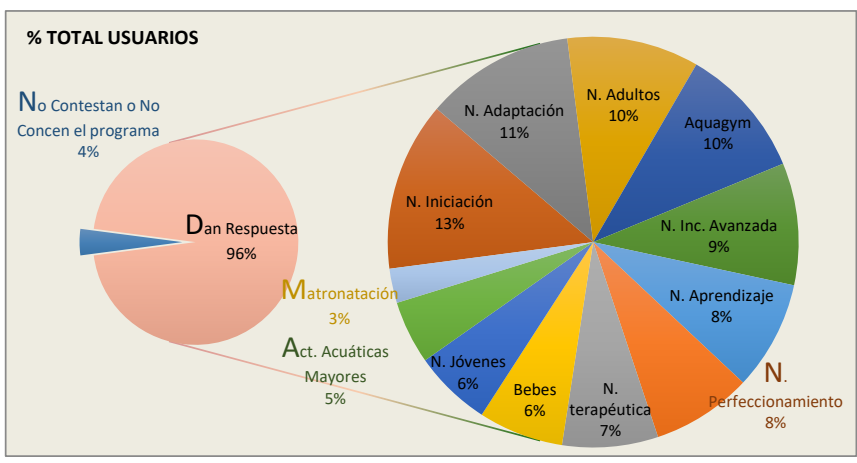

Figura 10. Actividades más conocidas por aquellos que no participan en programas acuáticos.

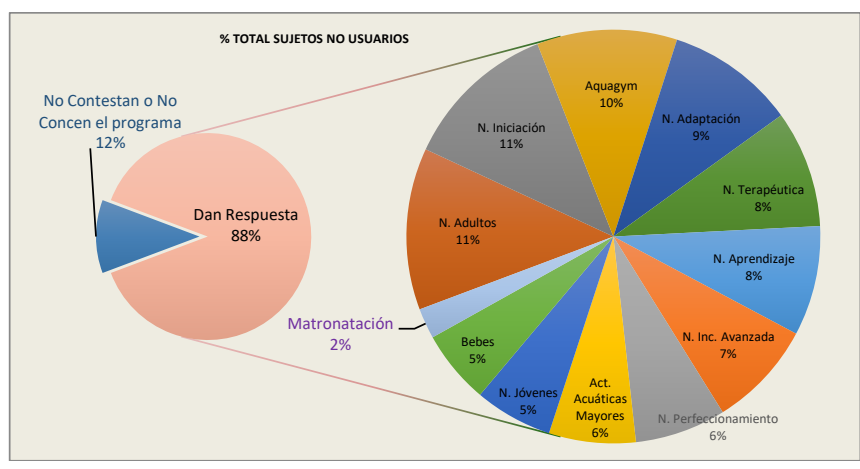

A continuación en las Figuras 11 y 12, se detalla la inclinación de la muestra en función del tipo de actividades que más interés les genera, siendo de estas, el fitness acuático la actividad más demandada en ambos grupos con un $35,6 \%$ de interés para los usuarios y un $44,7 \%$ para los no usuarios, en segunda instancia, el grupo de usuarios reclama la natación educativa con un $28,2 \%$ y deja en tercer y cuarto lugar las actividades relacionadas con aspectos de salud y rendimiento federado con un 17,6 y $17,4 \%$ respectivamente, para finalizar no prestando especial interés, a otro tipo de actividades con un 1,3\% (entrenamiento no federado, deportes acuáticos de equipo y natación libre). De forma similar ocurre con el grupo de sujetos que no participa en ningún programa ofertado, que deja en segundo y tercer lugar las actividades relacionadas con la salud con un $24,6 \%$ y la natación educativa con un $22,6 \%$ de interés, y anota el deporte federado y otro tipo de actividades con un 6,6 y $1,4 \%$ respectivos.

De igual forma en la tabla 2 se aprecia, en ambos casos, que, a la mayoría de los individuos participantes del estudio, encuentra interesante la posibilidad de programas abonados, 85 y $87 \%$, y el 15 y el $13 \%$ restantes, no consideran de su interés este tipo de ofertas.

En cuanto a la preferencia de días y franjas horarias de asistencia (Tabla 3 y Figura 13), la posible demanda latente de actividades acuáticas determina que los días más solicitados son los martes y jueves con un 19,4 y $20,2 \%$, seguidos de los lunes y miércoles con un 18,8 y $17,5 \%$, dejando los viernes con un interés o posibilidad de asistencia de un $12,7 \%$ y los sábados y domingos con un 7 y $4,4 \%$. Por otra parte, surgen tres grandes márgenes horarios de mayor interés, las franjas de 9 a $10 \mathrm{~h}(10,2 \%)$ y de 10 a 11 h (10,6\%) por las mañanas, de 16 a $17 \mathrm{~h}(9,8 \%)$ y de 17 a 18 h (12,2\%) por las tardes, y de 19 a 20 h $(7,5 \%)$ y de20 a $21 \mathrm{~h}(18,1 \%)$ por las tardes-noches.
Figura 11. Actividades de interés de usuarios.

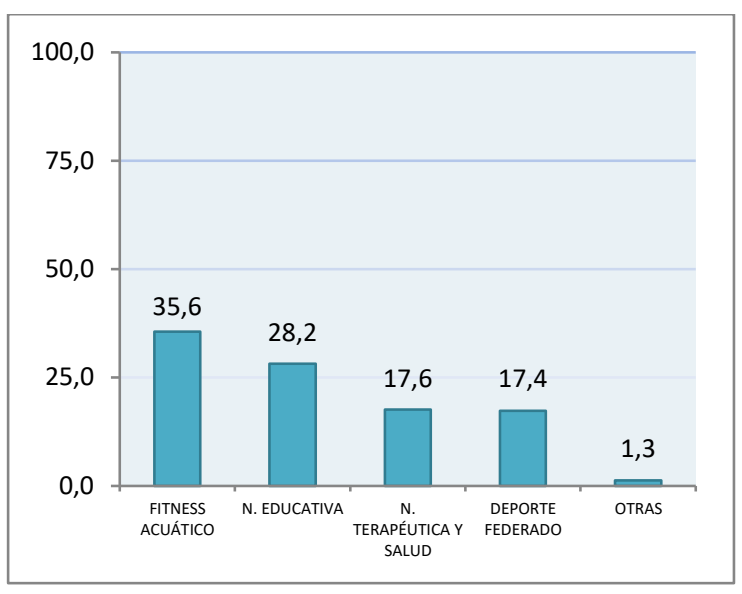

Figura 12. Actividades de interés de no usuarios.

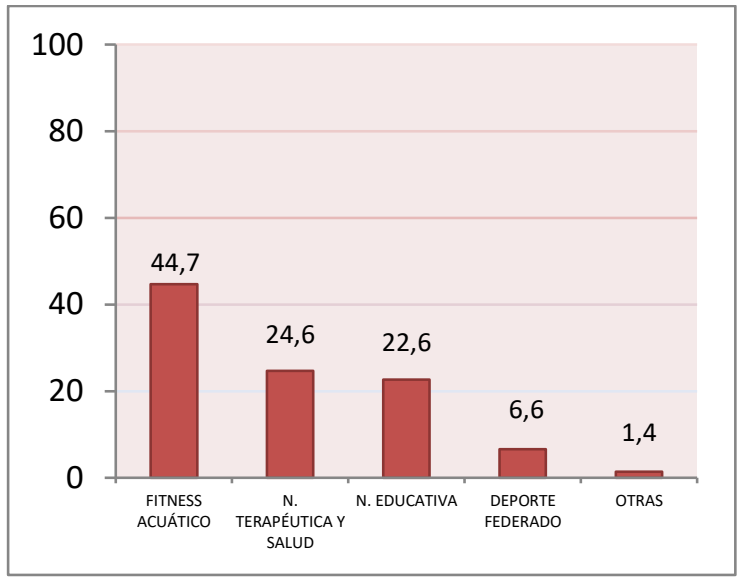

Tabla 2. Porcentaje de personas que consideran interesantes los programas de abono.

\begin{tabular}{|l|c|c|}
\hline & Interesados & No Interesados \\
\hline Usuarios & $84,8 \%$ & $15,2 \%$ \\
\hline No Usuarios & $87,3 \%$ & $12,7 \%$ \\
\hline
\end{tabular}

Figura 13. Distribución de días por interés marcado.

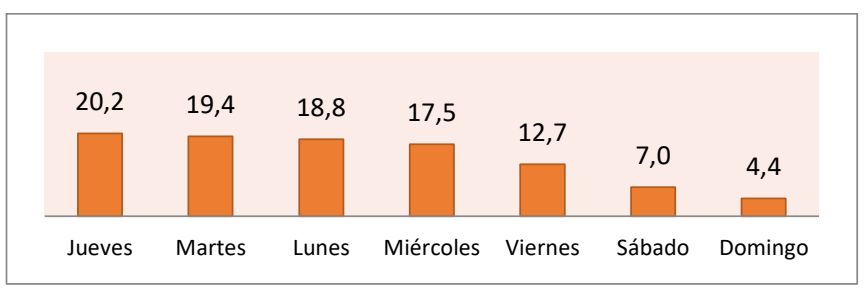

En el caso de los sujetos participantes en alguno de los programas, los motivos principales por los que acuden a estos son, por iniciativa propia con un $62,3 \%$, por recomendación de familias o amistades con un $17,3 \%$, un $9,8 \%$ por consejo médico, y un restante $11,5 \%$ distribuido en conocimiento de los programas a través de la administración local, de los medios de comunicación, por un evento deportivo, o por folletos y carteles (Figura 14). 
Tabla 3. Márgenes horarios de mayor interés.

\begin{tabular}{|l|c|l|c|}
\hline $\begin{array}{l}\text { Franja Horaria } \\
\text { Mañana }\end{array}$ & $(\%)$ & $\begin{array}{l}\text { Franja Horaria } \\
\text { Tardes }\end{array}$ & $(\%)$ \\
\hline 7:00 - 8:00 & 4,3 & $15: 00-16: 00$ & 0,8 \\
\hline 8:00 - 9:00 & 4,7 & $16: 00-17: 00$ & 9,8 \\
\hline 9:00 - 10:00 & 12,2 & $17: 00-18: 00$ & 12,2 \\
\hline 10:00 - 11:00 & 10,6 & $18: 00-19: 00$ & 4,7 \\
\hline 11:00 - 12:00 & 6,7 & $19: 00-20: 00$ & 7,5 \\
\hline 12:00 - 13:00 & 2,0 & $20: 00-21: 00$ & 18,1 \\
\hline 14:00 - 15:00 & 1,2 & $21: 00-22: 00$ & 5,1 \\
\hline
\end{tabular}

Figura 14. Distribución de los motivos por los que conocen el programa.

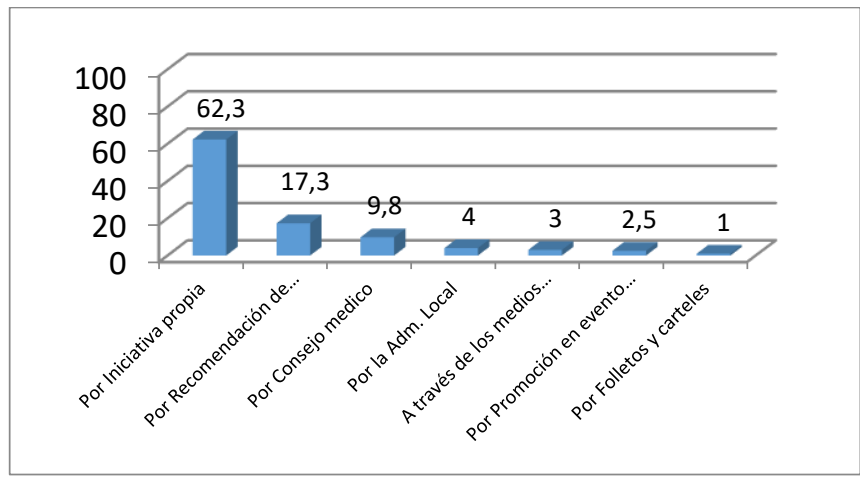

La satisfacción general de los usuarios asistentes a la piscina municipal queda expuesta en la figura 16, denotando gran satisfacción con el personal de la instalación, con una puntuación de 3,51 sobre cuatro puntos, dejando por detrás con la misma puntuación (2,73 puntos) la satisfacción con la instalación y sus accesos, y las deficiencias de la misma, y marcando una media de 2,59 puntos en la satisfacción de los usuarios con los vestuarios de esta.

En la Figura 15, además, se puede apreciar una disposición de las puntuaciones que dan los usuarios a sus monitores en cuanto al valor docente, marcando sobre cinco puntos, 4,18 para el desarrollo de la actividad por parte del monitor, 4,02 pts. para aspectos de resultado y evaluación y 3,97 pts. para la planificación de las sesiones.

Figura 15. Grado de satisfacción general.

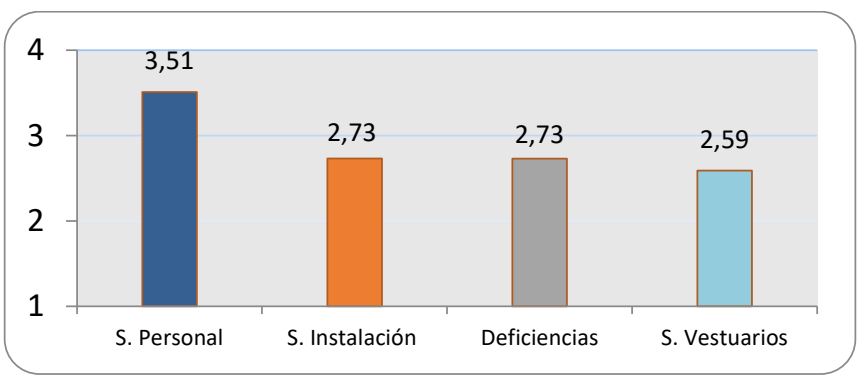

En lo referente a los motivos de práctica de ciertas actividades, ya sean acuáticas para los usuarios (Figura 17), o cualquiera para aquellos que no lo son (Figura 18), los objetivos que más se buscan a la hora de acudir a un programa, son, en primer lugar, aquellos relacionados con la salud tanto en usuarios (5,65 puntos sobre siete) como en no usuarios (5,91 pts.), en segundo lugar, el desarrollo de la habilidad con 5,27 puntos en los primeros y 4,95 puntos en los segundos, seguidos del tercer y cuarto puesto, que ocupan el pertenecer a un grupo social $(4,12$ pts.), y el aspecto físico y la imagen en los usuarios (3,63 pts.), y de forma inversa, con una media de 3,93 y 3,78 puntos, en los no usuarios, para finalizar con los aspectos que atienden a la relación social con 2,66 y 2,24 puntos respectivamente.

Figura 16. Evaluación docente.

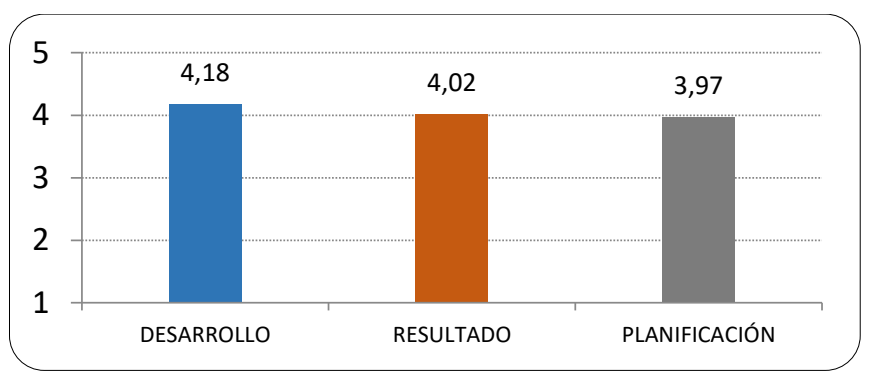

Figura 17. Motivos de práctica en actividades acuáticas.

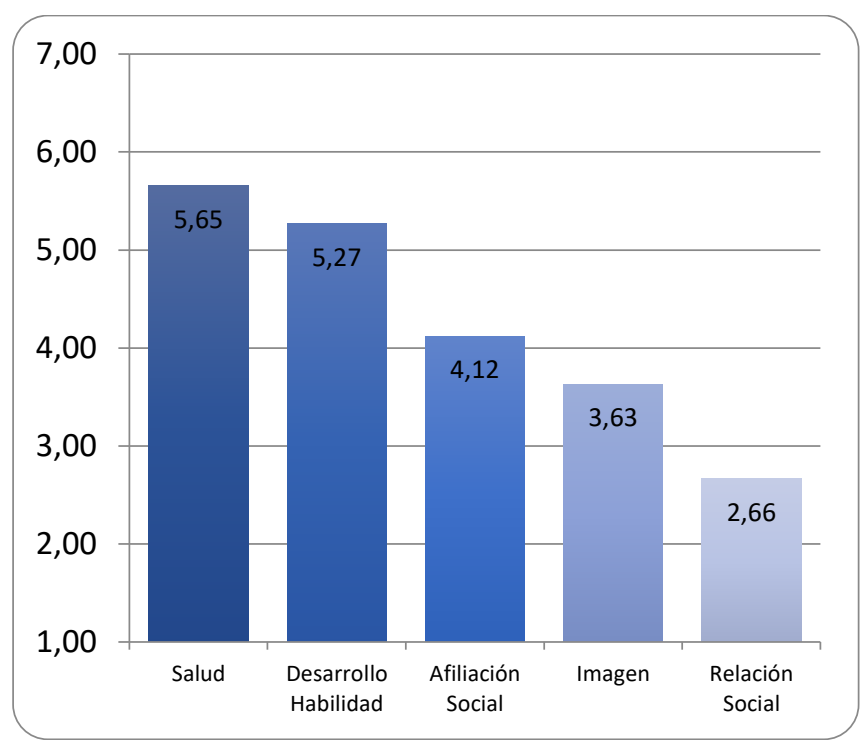

Figura 18. Motivos de práctica en ejercicio físico.

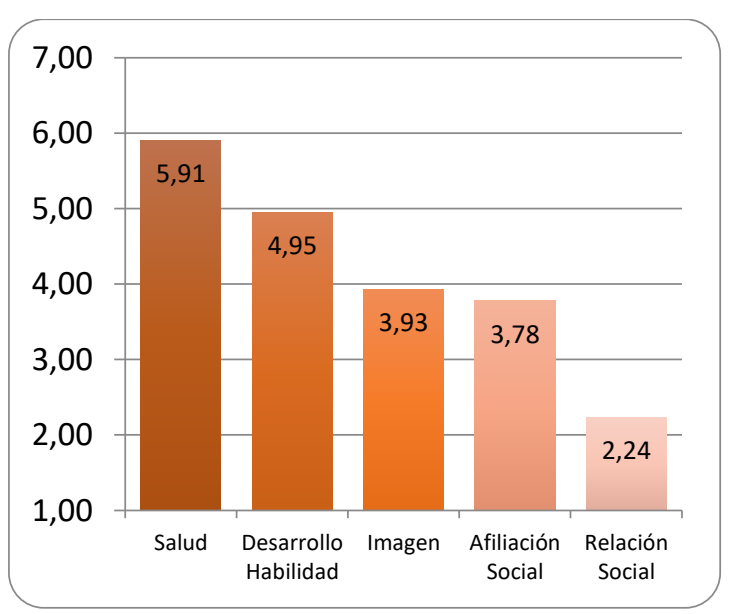

\section{Resultados diferenciales}

Según el género, en la Figura 19, podemos contemplar como las mujeres puntúan con 4,20 puntos el desarrollo de las actividades por parte de monitor, en similitud con los hombres que lo hacen con 4,14 pts., puntuando también algo por encima las mujeres (4,06 pts.) que los hombres (3,96 pts.) los aspectos relacionados con los resultados y la evaluación, para terminar con una puntuación muy igualada en la 
percepción de como planifica el monitor las clases $(3,98$ pts. las mujeres 3,95 y los hombres).

Además, según esta división por género, observamos que los aspectos que más satisfacen a ambos, son los relacionados con el personal de la instalación, siendo la media de puntuación para los hombres 3,55 y 3,49 para las mujeres. En cuanto al siguiente aspecto que mejor valoran, en el caso de las mujeres son los relacionados con la instalación $(2,8$ pts.) y en el de los hombres los vestuarios con 2,7 puntos, le siguen las deficiencias de la instalación como valor que tienen en cuenta con 2,78 pts. las mujeres y 2,64 los hombres, para terminar, valorando la instalación y sus espacios con 2,61 puntos los hombres, y las deficiencias con 2,53 las mujeres (Figura 20).

En lo relativo a los objetivos por los que ambos géneros se inscriben en programas acuáticos, podemos ver en la Figura 21, que los hombres puntúan algo por encima en todas las categorías, siendo estas puntuaciones para ellos: 5,82 en salud, 5,46 en desarrollo de la habilidad, 4,17 en afiliación social, 3,82 puntos de media en imagen, y 2,87 pts. para las relaciones sociales; y para las mujeres: 5,56 pts., 5,16 pts., 4,1 pts., 3,52 pts. y 2,55 puntos respectivamente.

Figura 19. Valor docente distribuido por sexo.

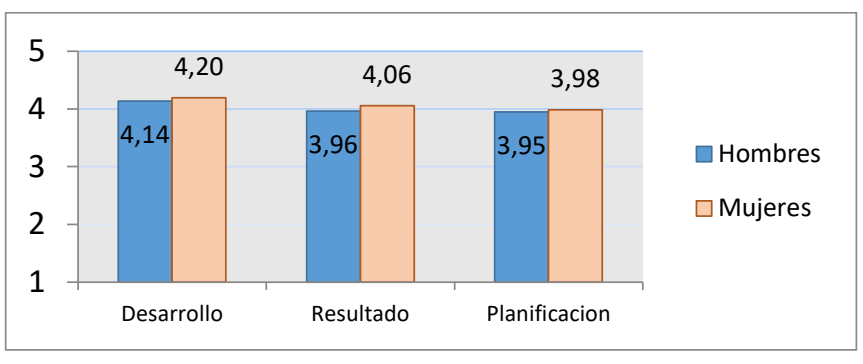

Figura 20. Satisfacción general en función del sexo.

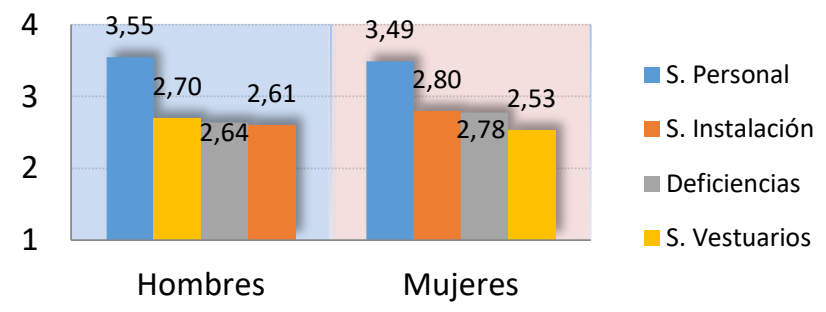

Figura 21. Motivos de práctica de actividades acuáticas por sexo.

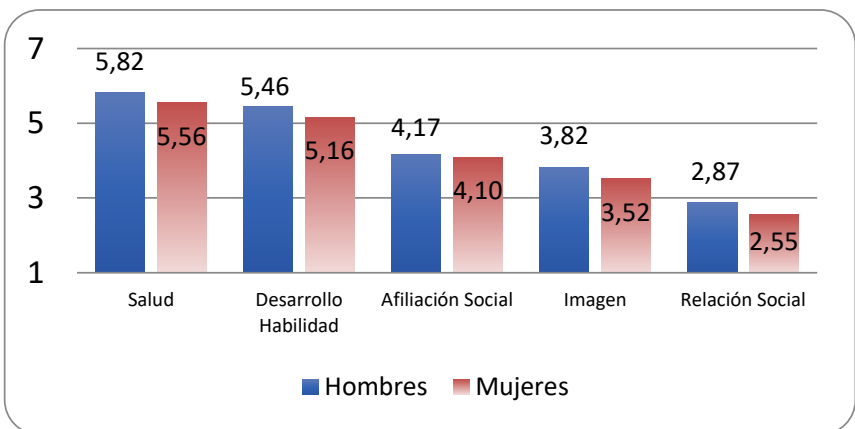

En cuanto al nivel de estudios, en la Figura 22 se aprecia una tendencia a puntuar el valor docente más bajo cuanto mayor es el nivel de estudios, quedando el desarrollo de la actividad con 4,46 para gente con estudios primarios, 4,13 para secundarios, y 4,07 puntos para estudios superiores, los aspectos relacionados con los resultados y evaluación del programa quedan distribuidos en 4,35 puntos para aquellos con estudios primarios, 4,01 con secundarios y 3,87 pts. para los que tienen estudios superiores. Quedando la planificación de las actividades con una percepción de 4,24 puntos para los primeros, 3,95 para los segundos, y 3,85 puntos para los terceros.

En esta comparativa, la satisfacción general con los aspectos relacionados con la instalación queda ordenada en: satisfacción con el personal con 3,73 puntos para estudios primarios. 3,49 para secundarios y 3,42 pts. de media para estudios superiores; satisfacción con la instalación y sus espacios, con 3,06 puntos, 2,73 puntos y 2,55 respectivamente; las deficiencias de la instalación la puntúan los estudios primarios con 2,92 pts., 2,77 puntos los secundarios, y 2,58 los superiores; y para terminar la satisfacción con los vestuarios queda repartida con unas medias de 2,72 pts., 2,56 y 2,55 puntos de forma pertinente (Figura 23).

Asimismo se mantienen el orden de los motivos principales de práctica por los que los usuarios se inscriben a estos programas, independientemente del nivel de estudios que presenten, quedando estos explicados (figura 25) con las siguientes medias: en motivos de salud con 5,46 para primarios, 5,61 en secundarios y 5,7 con estudios superiores; de igual forma 5,45, 5,11 y 5,33 puntos para desarrollo de la habilidad; afiliación social con 4,69, 4,08 y 3,86 puntos; de forma respectiva para los motivos de aspecto físico e imagen con 3,62, 3,61 y 3,65 puntos; y dejando para las relaciones sociales una puntuación de $2,98,2,68$ y 2,48 puntos de media.

Figura 22. Valor docente detectado por nivel de estudios.

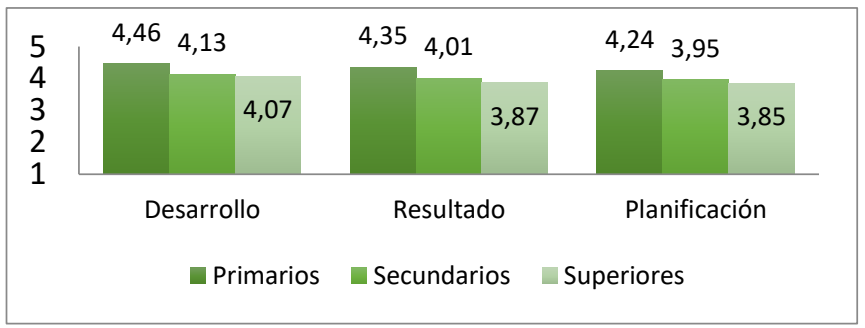

Figura 23. Satisfacción en función del nivel de estudios.

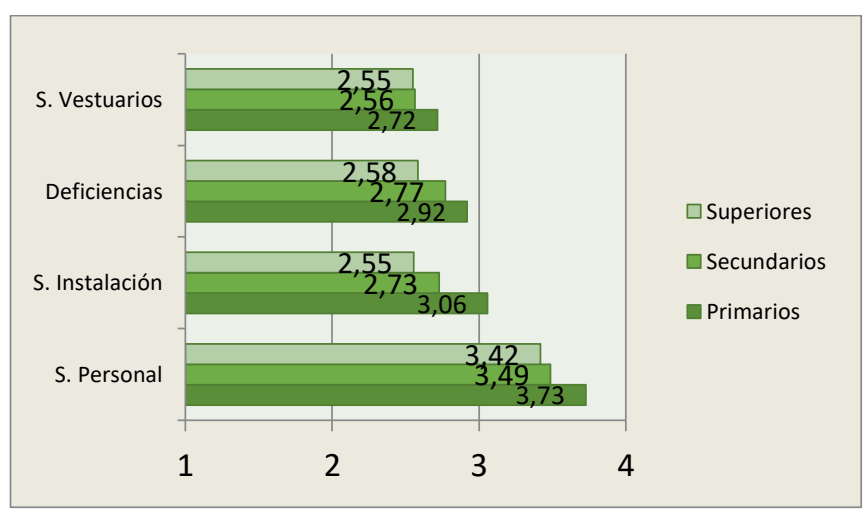

En última instancia, quedan expuestos los resultados atendiendo a los grupos de edad determinados para el estudio, dejando ver en la Figura 25 como se puntúa el valor docente del monitor de forma más estricta entre los dos rangos de edad intermedios, marcando los menores de 24 años, con una puntuación media de 4,2 los aspectos de desarrollo de la sesión, y 4,1 los de resultado y planificación de la misma. Seguidamente el rango de edad de 25 a 35 años puntúa el desarrollo de la sesión con 4 puntos, y el resultado y la planificación con 3,8 puntos cada uno. De forma similar puntúa el rango de los 36 a los 54 años, anotando unas puntuaciones de 4,1, 3,9 y 3,8 puntos respectivamente, para finalizar con las medias de puntuación que asignan los mayores de 55 años al desarrollo de la sesión (4,6 puntos), 
los resultados y la evaluación $(4,5)$ y la planificación (también 4,5 puntos).

Figura 24. Distribución de los objetivos de práctica según el nivel de estudios.

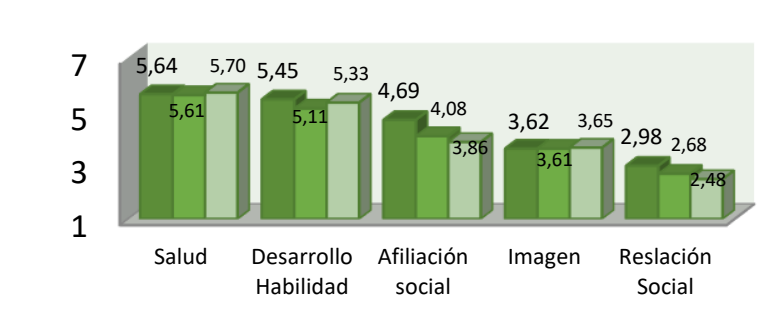

- Primarios $\square$ Secundarios $\square$ Superiores

Siguiendo con la misma dinámica, atendemos el nivel de satisfacción y como se ve afectado a lo largo de la edad en la Figura 26, apreciando que los menores de 24 años y mayores de 55 , puntúan por encima de los que están entre los 25 y 54 años, las características que afectan al servicio prestado en esta instalación. Siendo las puntuaciones de los menores de 24 años, 3,6 puntos en la satisfacción con el personal, y 2,8 para el resto de factores. En cuanto a los usuarios dados entre los 25 y 35 años, marcan una media de 3, 4 puntos para la satisfacción con el personal, 2,6 para la relativa a la instalación y sus espacios, y otros 2,6 y 2,5 puntos para las deficiencias y satisfacción con los vestuarios. Continuando con aquellos usuarios de entre 36 y 54 años que puntúan de la misma forma que los anteriores, exceptuando las deficiencias $(2,7$ puntos), para terminar con los valores dados por los mayores de 55 años, que son $3,9,3,2,3,1$ y 2,9 puntos respectivamente.

Por último, destacamos el progreso que hay en función de la edad, de los motivos por los que los usuarios realizan actividades acuáticas, dejando ver que los aspectos relacionados con la salud comienzan marcados por los menores de 24 años con 5,8 puntos de media y descienden de los 25 a los 35 años con 5,3 puntos, para luego aumentar en los siguientes rangos a 5, 6 entre los 35 y 54 años y 5,9 puntos para los mayores de 55 , siendo este motivo el más valorado por casi todas las franjas de edad. Lo mismo sucede con la afiliación social, en tercer lugar, que comienza con 4,6 puntos, descendiendo a 3,7 puntos, para luego subir a 4 y 4,7 puntos según los rangos de edad determinados. El segundo motivo más valorado (desarrollo de la habilidad) sufre un descenso de puntuaciones según avanzan los años, quedando en 5,9 para menores de 24 años, 5,1 entre los 25 y 35 años, 5,2 puntos para los que van de 35 a 54 años y 4,9 puntos para los mayores de 55. En el caso de la apariencia física, el valor dado en menores de 24 años es 3,8, para mantenerse durante el resto de edades en un 3,6, dejando este motivo en cuarto lugar, para terminar con los aspectos de relación social en último lugar como motivo de práctica, con unos valores de 3 puntos para la primera categoría, 2,3 para la segunda, 2,6 para la tercera, y 2,8 para la última categoría de edad (Figura 27).

Figura 25. Evaluación del monitor según la edad de los usuarios.

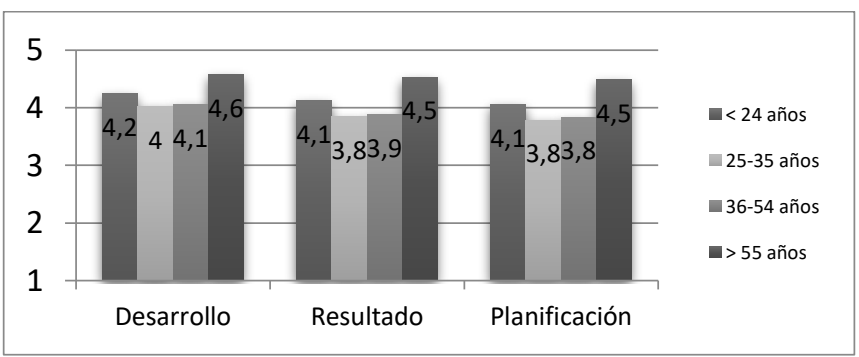

Figura 26. Nivel de satisfacción dado por franjas de edad.

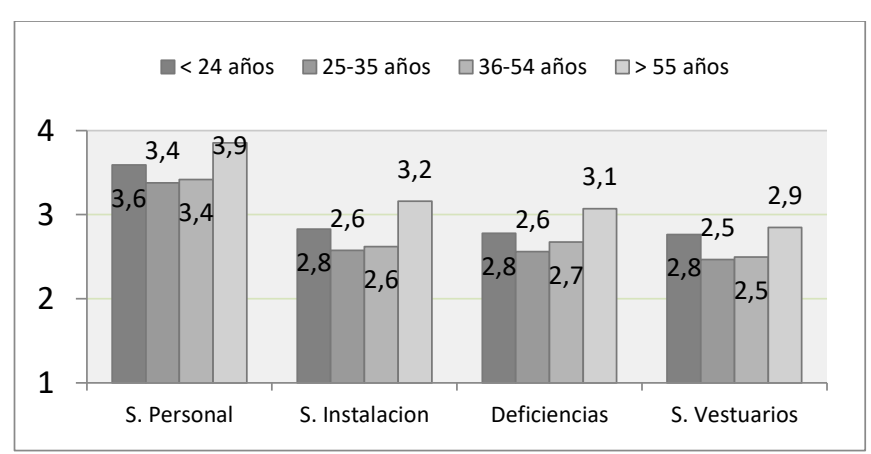

Figura 27. Motivos de práctica en función de la edad.

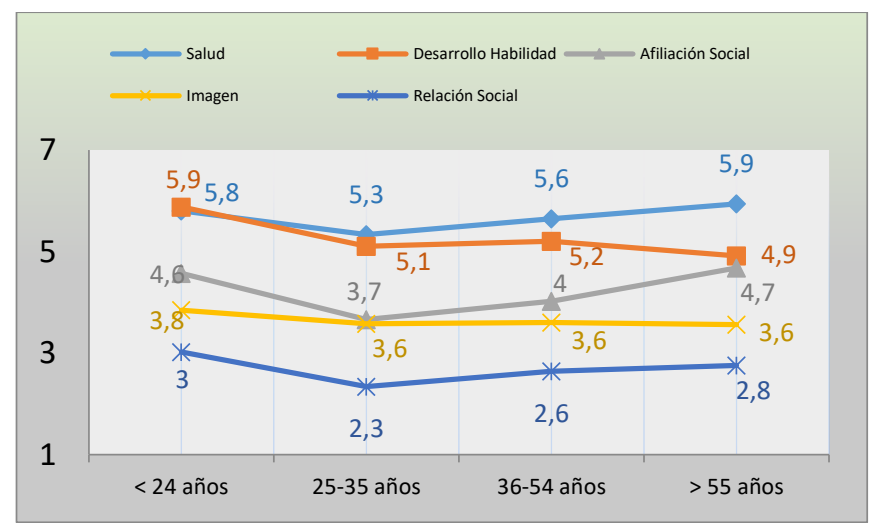

\section{Discusión}

De acuerdo a los objetivos fijados, abordamos en primer lugar los aspectos relacionados con la oferta de los servicios, y posteriormente aquellos que determinan los resultados de la demanda.

En relación a la satisfacción percibida con la instalación y los servicios de la misma, vemos que, coincidiendo con otros estudios como los de Armada et al. (2016), Nuviala, Tamayo y Nuviala (2012) y Sánchez et al. (2017), el personal que presta los servicios es el aspecto que mayor peso tiene en este análisis, en contraposición con los espacios y los vestuarios de la instalación que se llevan puntuaciones muy por debajo. Esto puede tener que ver con que, en cierta manera, los elementos tangibles adquieren en algunos casos las peores puntuaciones (Calabuig, Quintanilla y Mundina, 2008), o por otro lado, que en contraste con los resultados de Medina Rodríguez (2010), en los que define, según las consideraciones de los usuarios, al instructor de las actividades como un sujeto competente, atento, profesional, motivante y con dominio de su especialidad, este valor que se percibe en cuanto al personal, sea debido al aumento en la prioridad del nivel académico en las contrataciones, que ya se daba en 1998, y que según Álvarez-Fariña (2018), mencionaban Moreno y Gutiérrez en su manuscrito en aquellos años.

Manteniendo esta línea, vemos que, al aplicar una distinción en cuanto al género, se aprecian diferencias en los resultados. Al contrario que en nuestro estudio, que las mujeres puntuaron por debajo, Armada et al. (2016) y Medina Rodríguez (2010) destacan que, en su caso, encontraron diferencias significativas, siendo las mujeres las que más satisfacción percibían respecto a los hombres, sin embargo, en el documento de 2017 de Sánchez et al. podemos observar que no existían diferencias entre ambos sexos, dejando ver que cada contexto adquiere unos valores ajustados a lo que la situación determina. 
A parte de esto, si comparamos las franjas horarias de asistencia a los programas acuáticos de Moreno (1997), con las predilectas extraídas de nuestro análisis, podemos apreciar que hay cierta similitud, ampliando incluso una tercera franja a media tarde, en la que exponen sus intereses. Además, en el mismo documento, se confirman la falta de tiempo y los problemas laborales, como son la dificultad horaria y el malestar económico, como los principales motivos de no asistencia y abandono, refiriendo estos resultados con otros de estudios similares en su escrito.

En cuanto a las actividades que más demanda obtienen en este análisis, vemos que vienen en relación a lo que ya se ha extraído anteriormente de García-Ferrando y LLopis-Goig (2017), dejando claro que las actividades acuáticas están enmarcadas entre los deportes más practicados, y siendo el agua un medio totalmente adaptable a cualquier actividad que queramos trasladar, como bien exponen Colado, Moreno y Vidal (2001). Esto nos lleva a que podamos comparar resultados de preferencias de actividades en el ámbito general, como los de Martin et al. (2010), con los obtenidos en nuestro estudio, teniendo en cuenta que podrían darse actividades muy similares dentro del medio acuático.

Al igual que para este último autor, nuestros resultados determinan los motivos de salud como predilectos en cuanto a los beneficios que esperan obtener al acudir a este tipo de práctica, al contrario que los relacionados con la competencia y el desarrollo de la habilidad, que, en su caso, quedan por detrás de la búsqueda de beneficios sociales y ocupación del tiempo libre. Resultados similares obtuvieron MorenoMurcia, Marcos-Pardo y Huéscar (2016) que remarcan el auge que ha tenido la salud en la orientación de la práctica de actividad física en los últimos años, quedando también la salud como objetivo principalmente buscado, seguido de la búsqueda de mejorar la apariencia física y el desarrollo de la habilidad. En estudios algo más actuales se encontraron resultados idénticos a los nuestros, dejando ver un ascenso en la escala de los aspectos de autoeficacia a lo largo de estos años, posiblemente debido a la recompensa psicológica y emocional que supone lograr aquello que se han propuesto, ya que como confirman Borges-Silva, Prieto-Vaello, Alias y Moreno-Murcia (2015) existe una relación directa entre el motivo hacia la mejora de la salud y la motivación intrínseca y esto nos lleva a buscar un desarrollo en nuestra capacidad y querer mejorar nuestra habilidad (Guíu Carrera y Leyton Román, 2019), dejando en segundo plano, en estos casos, los cánones estéticos que anteriormente hacían buscar la mejora de la apariencia física al seguir los estereotipos establecidos por MorenoMurcia et al. (2016)

\section{Conclusión}

Atendiendo a la importancia de las actividades acuáticas en esta localidad e intentando comprobar la posibilidad de que estos programas respondan a los intereses reales de esta población, el objetivo tratado en este estudio fue el de analizar la oferta y la demanda de las actividades acuáticas llevadas a cabo en la misma, y así poder diferenciar que programas serían los idóneos para poner en práctica.

Tras el análisis de las variables sociodemográficas que definen tanto a los usuarios como a los no usuarios concluimos que:

- La mayor parte de la muestra de usuarios viene dada por usuarios de baño libre y participantes de actividades como iniciación a la natación, adaptación al medio acuático, natación para adultos y Aquagym, siendo la iniciativa propia principalmente, y la recomendación de amistades y el consejo médico como segundo y tercer motivo, lo que les mueve a acudir a las mismas.

- Casi el $80 \%$ de los sujetos no participantes en ningún programa acuático, realiza otras actividades uno o dos días a la semana principalmente y tres o más días como segunda y tercera opción, como pueden ser el Yoga o el Pilates, salir a correr, la gimnasia de mantenimiento y actividades relacionadas con el mundo del fitness, entre otras tantas menos destacadas.

- Los principales motivos por los que los sujetos no realizan ninguna actividad son la falta de tiempo, problema con los horarios, poco interés y los motivos económicos, dejando menos relevantes la lejanía de instalaciones y los motivos de salud.

- Más de la mitad de la muestra tanto en usuarios de programas como en los que no, son trabajadores, dedicándose entre un 30 y un $25 \%$ al estudio y en paro, entre otras.

- En ambos grupos, la mayoría de la muestra reside en la zona este y el centro urbano de la ciudad, quedando la zona oeste y las inmediaciones en segunda instancia algo menos participativas.

- Siendo la media mayoritaria de 2 hijos o ninguno, seguido de un $30 \%$ que tienen 1 o 3 hijos.

- Las actividades acuáticas más conocidas por los Yeclanos son las relativas a los más pequeños (entre los 3 y los 6 años) y la natación para adultos junto con el Aquagym. Quedando aun desconocimiento en una pequeña porción de la población del tipo de actividades ofertadas.

- El fitness acuático, principalmente, y la natación educativa son las actividades con mayor índice de interés registrado, quedando la natación terapéutica y el deporte federado con un interés relativo para los usuarios, y solamente la terapéutica para los no usuarios de programas.

- Hay un gran interés por la aplicación de programas abonados en los que puedan participar libremente, siendo los días más solicitados los martes y jueves, seguidos de lunes y miércoles, dejando los fines de semana con algo de interés en los viernes y solamente un $11 \%$ aproximado los sábados y domingos.

- Las mejores franjas para esta asistencia, queda determinada en 3 márgenes horarios uno por la mañana de 9 a 11, otro tras la comida de 16 a 18, y el último por la tarde-noche de 19 a 21 horas, siendo la franja más solicitada de 20 a $21 \mathrm{~h}$.

- En general, la satisfacción con el personal de la piscina es el aspecto que más se valora, seguido de una puntuación casi idéntica entre los niveles de satisfacción con la instalación actual y las deficiencias detectadas en la misma. En cuanto a los vestuarios, es el aspecto peor valorado en general, otorgándole menor puntuación el sexo femenino que el masculino, que este último, lo antepone a las deficiencias y la instalación.

- Se detecta una buena puntuación en cuanto a la forma en la que se imparten las clases, siendo el aspecto del desarrollo de la sesión en sí el que más puntuación logra, y dejando las evaluaciones y la planificación del programa como aspectos a poder mejorar en primer grado.

- Los principales motivos que mueven a los sujetos de ambos grupos hacia la práctica de actividad física son los relacionados con la salud y el desarrollo de la habilidad, seguidos del interés de pertenecer a un grupo y la mejora del aspecto físico. Esto viene marcado con más valoración por parte de los hombres que de las mujeres, y dando en varias ocasiones mayores puntuaciones cuantos menos estudios tengan, como en el caso del desarrollo de la habilidad, la pertenencia a un grupo y las relaciones sociales que son mejor valorados por los individuos con estudios primarios.

- En cuanto a la edad, se observa una tendencia general a ser más exigente en el rango de 25 a 54 años, especialmente en la satisfacción con los vestuarios, la instalación y las deficiencias existentes en la misma, siendo los mayores de 54 años los que mejor valoran en todos los casos. De igual forma ocurre con el personal que asiste sus clases, dejando ver que la franja de 24 años y especialmente los mayores de 54, valoran los 3 aspectos docentes por encima que la franja de edad intermedia.

- Igualmente ocurre con el nivel de estudios, cuanto más formación posee el individuo, mayor exigencia se aprecia y menor 
puntuación se registra, tanto en los niveles de satisfacción con la instalación y el personal, como la evaluación de la actuación del monitor en sus clases, dejando clara diferencia entre los menos formados, que apuntan por encima de los 4 puntos en los 3 aspectos docentes, y los sujetos con estudios superiores que apenas llegan a los 4 puntos en solo el aspecto del desarrollo, dejando los otros dos como aspectos más lejanos de la misma puntuación.

- Para los motivos de práctica, se observa una valoración menor en la franja de los 25 a los 35 años de todos los aspectos que llevan a la práctica de alguna actividad acuática, percibiendo también una tendencia a tener más en cuenta los motivos de salud y afiliación social conforme vamos creciendo, al contrario que el desarrollo de la habilidad, que comienza siendo el motivo más demandado y va disminuyendo conforme se hacen mayores, para terminar destacando que los motivos de imagen y relación social se mantienen más o menos lineales a lo largo de los años.

Por consiguiente y a raíz de todas estas diferenciaciones, se plantea se tengan en cuenta las siguientes ideas:

- Atendiendo a las actividades terrestres más demandadas por aquellos que no participan en ningún programa acuático, sería interesante plantear actividades acuáticas relacionadas con el Yoga, el Pilates o el Tai chí, gimnasia acuática de mantenimiento, fitness acuático e incluso actividades de carrera y cambios de ritmo en el medio acuático, con el fin de extrapolar los intereses de este colectivo, ofreciendo una alternativa acuática con numerosos beneficios y bajo impacto articular (Colado, Moreno y Vidal, 2001).

- De igual forma sería relevante el realizar los programas ajustando las actividades acuáticas a uno o dos días por semana, siendo el $54,9 \%$ del colectivo que práctica otro tipo de actividades, el hábito que más denota al elegir realizar una actividad.

- Considerando la falta de tiempo y la dificultad horaria, los motivos por los que mayoritariamente la posible demanda latente no asiste a ningún programa acuático, podría ser llamativo el realizar alguna actividad acuática aislada en días finales de semana, donde el tiempo generalmente suele ser más libre de ocupaciones.

- Teniendo en cuenta que más de la mitad de la población que contesta a la encuesta se encentra en situación laboral, y que dentro de los márgenes horarios más solicitados se encuentran un par de franjas (de 19 a 20 y de 20 a 21) que podrían permitir la asistencia de este sector, sugerimos que estas dos horas se planteen actividades acordes a sus intereses, siendo estos el fitness acuático y la natación educativa principalmente.

- Observando que gran parte de la población asistente a los programas queda aislada entre el centro y la zona oeste de la ciudad, quizás sería apropiado facilitar una línea de transporte que cubriera otros sectores igualmente extensos del municipio, como son la zona oeste y la alameda, que aunque más alejadas de la instalación, podrían ser un factor importante de demanda, siendo estos puntos un lugar de posible interés estratégico en el que acudieran individuos que residan en las inmediaciones de estas zonas.

- Prestando atención a las actividades ofertadas conocidas por los ciudadanos, se aprecia un relevante desconocimiento de gran parte de las actividades, principalmente las nuevas actividades como las de bebes y matronatación y otras que se salen del patrón de enseñanza en adultos, niños de entre 3 y 6 años, y Aquagym. Es por esto que proponemos se realicen de forma más asidua jornadas de puertas abiertas, días de acompañamiento por familiares o amigos en sesiones concretas como pueden ser las de bebes, matronatación y actividades para mayores o relacionadas con la salud, en las que, por el mayor control de la sesión, no supondría grandes problemas.
- Estudiando los días de mayor preferencia para asistir a cualquier programa deportivo, lo trasladamos al medio acuático, incitando a determinar las actividades mayormente demandadas en los días de lunes a jueves, y dejando los fines de semana como alternativa a programas de abono (con un interés de entre el 80 y $90 \%$ ) para aquellos que quieran completar un día más en su plan de ejercicio o incluso plantear actividades semanales de libre acceso, bajo previa inscripción, para estos tres últimos días.

- Igualmente apoyándonos en la preferencia de horarios de asistencia a cualquier actividad deportiva, definimos tres márgenes horarios puntuales, en los que deberían de darse las principales actividades demandadas. Estos son por la mañana de 9 a 10 y de 10 a $11 \mathrm{~h}$, por las tardes de 16 a 17 y de 17 a 18 horas, y por la tarde noche de 19 a 20 y de 20 a 21 horas.

- Atendiendo a los resultados obtenidos, se ve necesaria una especial atención a intentar depurar las posibles deficiencias de la instalación, especialmente en la zona de vestuarios y en particular el de las mujeres.

- En base a las respuestas dadas en relación a la actuación del monitor, sería de provecho centrar algo más de atención en la planificación y evaluación de los programas, principalmente en aquellos que impliquen las actividades con edades intermedias (25-54 años).

- Las actividades acuáticas enfocadas a los mayores de 54 años deberían ir encaminadas a motivos de salud y afiliación social, sin olvidar seguir desarrollando su habilidad, mientras que para los menores de 24 años principalmente debería buscarse esa mejora de habilidad y tener también en cuenta los aspectos de salud.

- Los principales programas en la instalación acuática, considerando los resultados, deberían estar enfocados principalmente al fitness acuático, seguido de actividades de enseñanza acuática educativa, y dando opciones de actividades como natación terapéutica y de rendimiento y perfeccionamiento. Siendo los objetivos principales que deberían desarrollar los de salud, seguidos de desarrollo de habilidad y continuados por aspectos de afiliación social y pertenencia al grupo. Un posible ejemplo podría darse de la siguiente forma, apoyado por Colado, Moreno y Vidal (2001) ya mencionados anteriormente:

a. Aquabuilding (actividades de musculación y trabajo de fuerza fundamentada en la resistencia que ofrece el agua, dando la posibilidad de alterar la intensidad modificando la velocidad de ejecución, posición articular, e incluso incorporando levantamiento de pesas), Aquagym (actividad multidisciplinar, variada y de trabajo moderado, en el que se realizan desde actividades de desplazamiento caminando, a ejercicios de fuerza, y pasando por movimientos con base rítmica hasta ejercicios aislados de natación) y GymSwim (disciplina que abarca una gran cantidad de actividades, basada en la selección ordenada y variada de ejercicios seleccionados en función de la lección a tratar y sustentada con un subfondo musical acorde con la sesión, pudiendo darse desde danza hasta boxeo en el agua) para programas de fitness acuático.

b. Aquastretching (dedicado a favorecer la movilidad articular y la elongación de la musculatura, haciéndose uso de la presión hidrostática y diferentes técnicas de estiramientos para fomentar la relajación y mejora de la salud) y Actividades de movilización de suelo pélvico y acondicionamiento de musculaturas estabilizadoras para usuarios que busquen la práctica de ejercicio por motivos de salud

c. Aquafic (programa específico de mejora de la condición física, estructurado en 4 niveles de condición, en el que se requiere de evaluación profesional con valores de prueba de esfuerzo y previa revisión médica que determinan en qué nivel comenzar y cuando poder avanzar al siguiente, se basa principalmente en entrenamiento interválico de moderada y alta intensidad) y programas de entrenamiento y perfeccionamiento técnico de 
los estilos de natación para aquellos que busquen la mejora de habilidad y el rendimiento expresamente.

Prospectivas de futuro:

- Buscando un mejor entendimiento de las variables que nos ocupan, serían necesarios más estudios con mayor número de muestra y con edades más concretas.

- Para futuros análisis, sería adecuado realizar el proceso en fechas algo más intermedias, como febrero o marzo, con el fin de evitar la celeridad y ganar la posibilidad de adquirir mayor número de muestra.

- Es interesante contemplar una posible reproducción y mejora del estudio en años venideros, para así poder comparar en el tiempo los cambios generados en la sociedad y determinar si las actuaciones pertinentes son las idóneas y van orientadas de forma acertada.

\section{Contribución e implicaciones prácticas}

Este estudio permite principalmente ahondar en descubrir los hábitos físicos deportivos en relación a las actividades en el medio acuático de la población de Yecla, lo que permite al órgano de gestión ajustar mejor la oferta de los servicios a la demanda extraída.

Proporciona un precedente, que posibilita comprobar en futuros análisis que el camino tomado es el correcto, aportando una base con la que contrastar la información a lo largo del tiempo, pudiendo encauzar las decisiones en función de las fluctuaciones apreciadas en los mismos.

El documento contribuye ampliando la literatura en relación a investigaciones similares.

\section{Agradecimientos}

Para poder llevar a cabo el texto, fue necesaria la colaboración y ayuda de instituciones como AIDEA y el Servicio Municipal de Deportes del ayuntamiento de Yecla, a las que le estoy muy agradecido por el apoyo prestado, y en especial este propósito no habría sido posible sin las valiosas dotes de paciencia y buena guía de un gran profesor, gracias por su excelencia.

\section{Referencias}

Álvarez-Fariña, R. (2018). Oferta de servicios y programas de natación y actividades acuáticas para población infantil mexicana. RIAA. Revista de Investigación en Actividades Acuáticas, 2(4), 77-81.

Armada, E., Martínez-Gallego, F., Segarra, E., \& Díaz, A. (2016). La satisfacción del usuario como indicador de calidad en el servicio municipal de deportes. Percepción, análisis y evolución. SPORT TK: Revista Euroamericana de Ciencias del Deporte, 5(1), 119-122.

Borges-Silva, F., Prieto-Vaello, A., Alias, A., \& Moreno-Murcia, J. A. (2015). Predicción del motivo salud en el ejercicio físico en centros de fitness. RICYDE. Revista Internacional de Ciencias del Deporte, 40(11), 163-172

Calabuig, F., Quintanilla, I., \& Mundina, J. (2008). La calidad percibida de los servicios deportivos: diferencias según, instalación, género, edad y tipo de usuario en servicios náuticos. Revista Internacional de Ciencias del Deporte, 10(4), 25-43.

Colado, J. C., Moreno, J. A., \& Vidal, J. (2001). Fitness acuático: una alternativa a las gimnasias de mantenimiento. Apunts: Educación Física y Deportes, 62, 68-79.

García Ferrando, M., \& Llopis Goig, R. (2017). La popularización del deporte en España: encuestas de hábitos deportivos 19802015 (Vol. 50). Madrid: CIS-Centro de Investigaciones Sociológicas.
García, J., Vergara, J., López, G., \& Díaz, A. (2016). Satisfacción de usuarios de servicios deportivos en Orihuela (Alicante). SPORT TK: Revista Euroamericana de Ciencias del Deporte, 5(Supl.), 155-162.

Guíu Carrera, M., \& Leyton Román, M. (2019). Perfil psicológico en corredores de ultramaratón (Psychological profile in ultramarathon runners). Retos, 36(36), 310-317.

Martín, M., González, M. D., Campos, A., Del Hierro, D., \& JiménezBeatty, J. E. (2010). Expectativas en la demanda latente de actividad física de las mujeres y hombres mayores en España. Cultura, Ciencia y Deporte, (15), 141-150.

Medina Rodríguez, R. (2010). Opinión de los usuarios/deportistas acerca de los servicios ofrecidos por una entidad deportiva municipal. Revista Mexicana de Investigación en Cultura Física y Deporte, 2(2), 236-252.

Ministerio de Educación, Cultura y Deporte. Encuesta de hábitos deportivos en España, 2015. España: Catalogo de publicaciones del ministerio. Recuperado de http://www.culturaydeporte.gob.es/servicios-alciudadano/estadisticas/deportes.html

Morales, F., \& Zárate, M. A. (2017). Turismo de interior y desarrollo local sostenible en Yecla. Yecla: Excmo. Ayuntamiento de Yecla, Instituto de Turismo de la Región de Murcia y Real Sociedad Geográfica.

Morales Yago, F. J. (2006). Consecuencias de la globalización en el espacio urbano y la dinámica poblacional en Yecla. Yecla: Excmo. Ayuntamiento de Yecla y Colegio "La Inmaculada".

Moreno, J. A. (1997). Relación oferta-demanda de las instalaciones acuáticas cubiertas: bases para un programa motor en actividades acuáticas educativas. Tesis doctoral. Dir. Dr. Melchor Gutiérrez. Facultad de Psicología. Universidad de Valencia.

Moreno-Murcia, J. A., Marcos-Pardo, P. J., \& Huéscar, E. (2016). Motivos de Práctica Físico-Deportiva en Mujeres: Diferencias entre Practicantes y no Practicantes. Revista de Psicología del Deporte, 25(1), 35-41.

Moreno-Murcia, J. A., Silveira, Y., \& Belando, N. (2015). Questionnaire evaluating teaching competencies in the university environment. Evaluation of teaching competencies in the university. New Approaches in Educational Research, 4(1), 54-61.

Nuviala A., Tamayo J., y Nuviala, R. (2012). Calidad percibida del deporte escolar como predictor del abandono deportivo en adolescentes. Revista Internacional de Medicina y Ciencias de la Actividad Física y el Deporte, 12(47), 389-404.

Sánchez, C., González, C., López, G., \& Díaz, A. (2017). Satisfacción de clientes externos: Estudio de caso de una piscina cubierta. SPORT TK: Revista Euroamericana de Ciencias del Deporte, 6(2), 81-88.

Sebire, S. J., Standage, M., \& Vansteenkiste, M. (2008). Development and validation of the Goal Content for Exercise Questionnaire. Journal of Sport and Exercise Psychology, 30, 353-377.

Tuero, C., \& González-Boto, R. (2015). Los usuarios de equipamientos acuáticos: aportaciones al ámbito de la gestión deportiva. Apunts. Educación física y deportes, 3(121), 64-72. 